\title{
The evolution of chemical abundance in quasar broad line region
}

\author{
Fei Xu, ${ }^{1,2 \star}$ Fuyan Bian, ${ }^{2,3 \star}, \dagger$ Yue Shen, ${ }^{4}$ Wenwen Zuo, ${ }^{5}$ Xiaohui Fan ${ }^{6}$ and \\ Zonghong $\mathrm{Zhu}^{1}$ \\ ${ }^{1}$ Department of Astronomy, Beijing Normal University, 19 Xinjiekou Outer St, BeiTaiPingZhuang, Haidian Qu, Beijing 100875, China \\ ${ }^{2}$ Research School of Astronomy and Astrophysics, Mt Stromlo Observatory, Australian National University, Canberra, ACT 2611, Australia \\ ${ }^{3}$ European Southern Observatory, Alonso de C'ordova 3107, Casilla 19001, Vitacura, Santiago 19, Chile \\ ${ }^{4}$ Department of Astronomy, University of Illinois Urbana-Champaign, 1002 W. Green Street Urbana, IL 61801, USA \\ ${ }^{5}$ Shanghai Astronomical Observatory, Chinese Academy of Sciences, 80 Nandan Rd., Shanghai 200030, China \\ ${ }^{6}$ Steward Observatory, University of Arizona, 933 N Cherry Ave, Tucson, AZ 85719, USA
}

Accepted 2018 July 1. Received 2018 May 25; in original form 2017 July 31

\begin{abstract}
We study the relation between the metallicity of quasar broad line region (BLR) and black hole (BH) mass $\left(10^{7.5}-10^{10} \mathrm{M}_{\odot}\right)$ and quasar bolometric luminosity $\left(10^{44.6}-10^{48} \mathrm{erg} \mathrm{s}^{-1}\right)$ using a sample of $\sim 130000$ quasars at $2.25 \leq z \leq 5.25$ from Sloan Digital Sky Survey Data Release 12 (DR12). We generate composite spectra by stacking individual spectra in the same BH mass (bolometric luminosity) and redshift bins and then estimate the metallicity of quasar BLR using metallicity-sensitive broad emission-line flux ratios based on the photoionization models. We find a significant correlation between quasar BLR metallicity and BH mass (bolometric luminosity) but no correlation between quasar BLR metallicity and redshift. We also compare the metallicity of quasar BLR and that of host galaxies inferred from the massmetallicity relation of star-forming galaxy at $z \sim 2.3$ and 3.5. We find quasar BLR metallicity is $0.3 \sim 1.0$ dex higher than their host galaxies. This discrepancy cannot be interpreted by the uncertainty due to different metallicity diagnostic methods, mass-metallicity relation of galaxy, metallicity gradient in quasar host galaxies, BH mass estimation, the effect of different spectral energy distribution models, and a few other potential sources of uncertainties. We propose a possibility that the high metallicity in quasar BLR might be caused by metal enrichment from massive star formation in the nucleus region of quasars or even the accretion disc.
\end{abstract}

Key words: galaxies: abundances - galaxies: active - galaxies: high redshift - quasars: emission lines.

\section{INTRODUCTION}

Quasars are the most luminous subclass of active galactic nuclei. They provide powerful tools to study the re-ionization process (Fan, Carilli \& Keating 2006a; Fan et al. 2006b), supermassive BH (SMBH) growth (Mortlock et al. 2011; Wu et al. 2015), and chemical enrichment history (Hamann et al. 2002; Dietrich et al. 2003; Nagao, Marconi \& Maiolino 2006) at the early epoch of the Universe. Quasars are powered by accreting material on to the central SMBHs. The BLRs contain gas clouds close to the SMBHs, which are photoionized by the radiation field from the accretion disc of the SMBH. The ultraviolet and optical emission lines from BLRs are widely used to estimate the SMBH mass (Vestergaard \& Peterson 2006; Shen 2013; Zuo et al. 2015) and the chemical

\footnotetext{
^E-mail: astroxf@163.com (FX); Fuyan.Bian@eso.org (FB)
}

$\dagger$ Stromlo Fellow. abundance close to the SMBH (Hamann et al. 2002; Nagao et al. 2006; Jiang et al. 2007; Juarez et al. 2009; Matsuoka et al. 2011; Marziani et al. 2015).

Studies on the chemical abundance in the SMBH BLR provide insights on the chemical evolution of their host galaxies and shed light on the co-evolution of galaxies and their central SMBHs. The gas-phase metallicity in the BLR can be measured from broad emission-line flux ratios. Robust metallicity-indicating line ratios, such as $\mathrm{Nv} / \mathrm{C}$ Iv, $\mathrm{N}$ v/He II, can be used to estimate metallicity by using the photoionization models (Hamann et al. 2002; Nagao et al. 2006).

The measurements of the metallicity in quasar BLR have led to three intriguing findings: (1) Super solar metallicity in the BLR of luminous quasars at high redshift. Dietrich et al. (2003) found that the metallicity of quasar BLR is at least four times of the solar metallicity $\left(\mathrm{Z}_{\odot}\right)$ in 70 most luminous $z \geq 3.5$ quasars. These authors suggested intense star formation in quasar host galaxies 
at the early epoch of the Universe to enrich the quasar BLR. (2) There exists a strong correlation between the metallicity of quasar BLR and SMBH mass as well as quasar luminosity (e.g. Warner, Hamann \& Dietrich 2003; Nagao et al. 2006; Matsuoka et al. 2011). (3) However, the metallicity of quasar BLR does not evolve with cosmic time (Warner et al. 2003; Nagao et al. 2006).

The evidence that there is no redshift evolution of the chemical abundance in the quasar BLR is quite puzzling. Studies have found a strong metallicity evolution in galaxies over cosmic time. Highredshift star-forming galaxies have lower gas-phase metallicity than their low-redshift counterparts for a given stellar mass (Erb et al. 2006; Maiolino et al. 2008; Zahid et al. 2013; Ly et al. 2014; Maier et al. 2014; Steidel et al. 2014; Salim et al. 2015; Sanders et al. 2015; Guo et al. 2016; Onodera et al. 2016).

One interpretation of the non-evolution of metallicity at different redshifts is that the quasars in the above studies are biased to the most luminous quasars which are hosted by the most massive galaxies for a given redshift. Given the fact that the evolution of the galaxy mass-metallicity becomes weaker towards the high-mass end (Maiolino et al. 2008; Zahid et al. 2013; Onodera et al. 2016), the metallicities in these luminous quasars may not evolve dramatically across cosmic time as well. Therefore, it is essential to study the chemical abundance in quasars with a broad range of luminosity, $\mathrm{BH}$ mass, and redshift.

To solve the above issues, we select a sample of $\sim 130000$ quasars in the redshift range of $2.25 \leq z \leq 5.25$ from the Sloan Digital Sky Survey (SDSS) Data Release 12 (DR12; Alam et al. 2015). The BH mass range of this sample is $10^{7.5} \leq M_{\mathrm{BH}} \leq 10^{10} \mathrm{M}_{\odot}$ and the bolometric luminosity range is $10^{44.2} \mathrm{erg} \mathrm{s}^{-1} \leq L_{\text {bol }} \leq 10^{48} \mathrm{erg} \mathrm{s}^{-1}$. We divide this quasar sample into different redshift and $\mathrm{BH}$ mass (bolometric luminosity) bins and generate the corresponding composite spectra in order to investigate the relationship between metallicity and BH mass (bolometric luminosity) at different redshifts. This sample is the largest quasar sample used to study the mass (bolometric luminosity)-metallicity relation. With this large sample and the composite spectra with a high signal-to-noise ratio $(\mathrm{S} / \mathrm{N})$, we can get a precise measurement of line ratio to estimate the metallicity of quasar BLR and then get a better statistical investigation for the evolution of metallicity in a wide range of $\mathrm{BH}$ mass and bolometric luminosity. We mainly focus on the metallicity of BLR in this work instead of narrow line region (NLR) which is supposed to trace the spatial scale (Bennert et al. 2006a,b) and the enrichment history of quasar host galaxies (Nagao et al. 2010). This is because that some typical NLR metallicity indicators, like $[\mathrm{N}$ II] $/ \mathrm{H} \alpha$ (Ludwig et al. 2012; Du et al. 2014), have lines that exceed the wavelength coverage of SDSS spectra, especially at higher redshift. Due to this problem, it is not easy to do sufficient statistical analysis on NLR with a wide redshift range comparing to BLR. The metallicity in quasar BLR can be affected by local starbursts at the centre of quasar host galaxies (Nagao et al. 2010), so it may not well present the global metallicity in host galaxies. But comparing the metallicity in the quasar BLR and its host galaxies can provide crucial information on the different history of star formation and metallicity enrichment in different parts of the galaxies.

This paper is organized as follows. In Section 2, we describe how we select quasars from the SDSS data and generate composite spectra in each of the BH mass (bolometric luminosity) and redshift bins. In Section 3, we describe the fitting method of broad emission lines, the metallicity measurement, and the corresponding results. We compare the metallicity in the quasar BLRs with that in the quasar host galaxies and present some discussion and possible explanations on the discrepancy between them in Section 4. We summarize our main conclusions in Section 5. We adopt $\left(\Omega_{\mathrm{tot}}, \Omega_{\mathrm{M}}\right.$, $\left.\Omega_{\Lambda}\right)=(1.0,0.3,0.7), \mathrm{H}_{0}=70 \mathrm{~km} \mathrm{~s}^{-1} \mathrm{Mpc}^{-1}$ (Spergel et al. 2007), and solar oxygen abundance of $12+\log (\mathrm{O} / \mathrm{H})=8.69$ (Asplund et al. 2009) in this paper.

\section{SAMPLE AND COMPOSITE SPECTRA}

\subsection{Sample selection}

We select quasars from the SDSS DR12 quasar catalogue (Pâris et al. 2017). There are 297301 quasars in the SDSS DR12 quasar catalogue. Most of the quasars in SDSS DR12 are observed as part of SDSS/BOSS quasar survey. The detail of the target selection can be found in Ross et al. (2012). We adopt redshift from visual inspection (Z_VI) in SDSS DR12 quasar catalogue and we refer to readers Pâris et al. (2017) for details of their visual inspection process. We estimate the virial $\mathrm{BH}$ mass of DR12 quasars by using the calibration from Vestergaard \& Peterson (2006) (VP06). The equation is as follows:

$$
\begin{aligned}
\log \left(\frac{M_{\mathrm{BH}}}{\mathrm{M}_{\odot}}\right)= & 0.66+0.53 \log \left(\frac{\lambda L_{1350 \AA}}{10^{44} \mathrm{erg} \mathrm{s}^{-1}}\right) \\
& +2 \log \left(\frac{\mathrm{FWHM}_{\mathrm{C} \mathrm{IV}}}{\mathrm{km} \mathrm{s}^{-1}}\right) .
\end{aligned}
$$

The full width at half-maximum (FWHM) of C IV emission line is from Pâris et al. (2017) and we exclude all the quasars whose FWHM_C IV is equal to -1 , which means $\mathrm{C}_{\mathrm{IV}}$ is not in the spectra. We define several line-free windows: $1350-1360 \AA, 1445-1455 \AA$, 1700-1705 A, 1770-1800 A, 2155-2400 A, 2480-2675 A, 1150 $1170 \AA, 1275-1290 \AA$, and $2925-3400 \AA$, to fit the power-law continuum and then estimate the monochromatic flux at $1350 \AA$.

We estimate the bolometric luminosity of DR 12 quasars by using the monochromatic flux at $1350 \AA$ and a bolometric correction of 3.81 (Richards et al. 2006).

Then, we use the following criteria to select quasars: (i) We exclude quasars with broad absorption lines (BALs) in the SDSS DR 12 quasar catalogue using BAL_FLAG_VI, which were determined by visual inspection, because BAL will affect the measurement on line flux and might also introduce uncertainty to the FWHM. Quasars with FWHM_C IV equal to -1 are also excluded. A total of 193768 quasars are left after this step. (ii) The redshift of selected quasars is between 2.25 and 5.25. Most UV metallicity diagnostic lines (e.g. $\mathrm{N} v, \mathrm{C}$ IV) fall into SDSS spectroscopic wavelength coverage ${ }^{1}$ within this redshift range. We only include quasars with ZWARNING $=0$, which means that the pipeline redshift is very reliable. According to Pâris et al. (2017), the identification or redshift of 3.3 per cent of these objects with ZWARNING $=0$ changed after visual inspection. For the remaining 96.7 per cent, the redshifts from visual inspection are same with the pipeline redshifts. 17 per cent of the overall SDSS samples have ZWARNING $>0$, which means that the pipeline output may not be reliable. For these objects, only 41.1 percent of the identification or redshift changed after visual inspection. Others are still ZWARNING $>0$. We exclude the quasars with ZWARNING $>0$ in order to make sure the redshift measurement of our sample is secure enough. A total of 133374 quasars are left after this step. (iii) We exclude quasars whose $\mathrm{BH}$ masses are not in the range of $10^{7.5} \mathrm{M}_{\odot} \leq M_{\mathrm{BH}} \leq 10^{10.0} \mathrm{M}_{\odot}$ and bolometric luminosity is not in the range of $10^{44.2} \mathrm{erg} \mathrm{s}^{-1} \leq L_{\text {bol }} \leq 10^{48.0} \mathrm{erg} \mathrm{s}^{-1}$. Only a few quasars are out of this range and they unevenly scatter in a

\footnotetext{
${ }^{1}$ The SDSS/BOSS wavelength coverage is from 3600 to $10500 \AA$.
} 
relatively wide $\mathrm{BH}$ mass or bolometric luminosity section, which makes it hard to divide them into bins, so we exclude them. Please see Fig. 1 for the details.

Given these constraints on the SDSS DR12 quasar catalogue, we select $\sim 130000$ quasars as our final sample to investigate the relationship between BH mass (bolometric luminosity) and quasar BLR metallicity at different redshifts.

\subsection{Composite spectra}

The main purpose of this work is to study the relationship between quasar BLR metallicity and BH mass (bolometric luminosity) at different redshifts. This requires us to measure the broad emission lines of quasar spectra in order to get the quasar BLR metallicity. Unfortunately, the individual SDSS spectra are too noisy to detect weak and highly blended metallicity diagnostic emission lines. Thus, we study the metallicity in quasar BLRs by stacking quasar spectra in the same redshift and $\mathrm{BH}$ mass (bolometric luminosity) bins.

We divide our sample into several redshift, $\mathrm{BH}$ mass, and bolometric luminosity bins. Tables $1-4$ summarize the redshift and BH mass (bolometric luminosity) bins and the number of quasars in each of the bins. Most bins have enough quasars for statistics and the sample is uniformly distributed in each bin as much as possible.

We generate the corresponding composite spectrum in each bin as follows:

(i) We obtain the reduced one-dimensional quasar spectra from the SDSS DR12.

(ii) We correct Galactic extinction using the Schlegel, Finkbeiner \& Davis (1998) map and Milky Way extinction curve (Cardelli, Clayton \& Mathis 1989).

(iii) We mask out $5 \sigma$ outliers below the 20-pixel boxcarsmoothed spectrum to reduce the effects of narrow absorption (Shen et al. 2011). Bad pixels are also masked out.

(iv) We shift each of the observed SDSS spectra into the rest frame based on the visual redshift.

(v) We re-sample each of the spectra with $1.0 \AA$ bins into a unified wavelength range from 1000 to $2000 \AA$ with a flux conservative manner for all redshift bins in range $2.25<z<3.75$. The wavelength range is from 1000 to $1800 \AA$ for quasars at $3.75<z<4.25$ and 1000 and $1600 \AA$ for quasars at $4.25<z<4.75$ and $4.75<z<$ 5.25 , due to the poor sensitivity beyond $10000 \AA$. This rest-frame wavelength range covers all the lines used to estimate metallicity.

(vi) Because we are more interested in the emission line properties but not the continuum of the quasar spectra, we calculate the arithmetic mean flux instead of the geometric mean at each wavelength pixel to generate the composite spectra (Vanden Berk et al. 2001). We derive the errors of the composite spectra by using the Monte Carlo simulation (Jones, Stark \& Ellis 2012). We first randomly choose 90 per cent of total individual spectra for a given $\mathrm{BH}$ mass (bolometric luminosity) and redshift bin and generate a 'fake' composite spectrum by following the above steps from (i) to (v). We repeat this for 1000 times and generate 1000 'fake' composite spectra. We then calculate the standard deviation of these 'fake' composite spectra at each wavelength pixel and take them as the uncertainties of the real composite spectrum.

These high S/N ( 100 per resolution element) composite spectra enable us to detect the weak emission lines to measure the quasar BLR metallicity. However, we exclude several composite spectra due to their relatively poor $\mathrm{S} / \mathrm{N}(\sim 10$ per resolution element). Please see Tables $1-4$ for more details.

\section{METALLICITY MEASUREMENT}

\subsection{Emission-line measurement}

We fit the continuum of quasar composite spectra using a powerlaw function. We use the following line-free wavelength regions, $1445 \AA<\lambda<1455 \AA$ and $1973 \AA<\lambda<1983 \AA$, for the continuum fitting of composite spectra in the redshift bins 2.25-2.75, 2.75-3.25, and3.25-3.75 (Nagao et al. 2006; Matsuoka et al. 2011). We use wavelength regions $1445 \AA<\lambda<1455 \AA$ and $1687 \AA<$ $\lambda<1697 \AA$ (Nagao et al. 2006) for composite spectra in redshift bin $3.75-4.25$, and $1275 \AA<\lambda<1290 \AA$ and $1445 \AA<\lambda<1455 \AA$ for composite spectra in redshift ranges $4.25-4.75$ and $4.75-5.25$ for power-law fitting because some line-free windows at longer wavelength have exceeded the coverage of SDSS spectrum at high redshift.

Two methods are widely used to measure line flux (Nagao et al. 2006). One is by fitting the line with Gaussian function or Lorentzian function (Zheng et al. 1997). The other one is by integrating the line flux above a defined continuum flux without fitting the line profile (Vanden Berk et al. 2001). Both approaches have limitation, especially when they are used to measure lines that are heavily blended with each other (e.g. Ly $\alpha, \mathrm{N} v$ ). In this study, we adopt the method from Nagao et al. (2006) and Matsuoka et al. (2011) to fit the emission line by the following function:

$\mathrm{F}_{\lambda}=\left\{\begin{array}{ll}\mathrm{F}_{0} \times\left(\frac{\lambda}{\lambda_{0}}\right)^{-\alpha} & \lambda>\lambda_{0} \\ \mathrm{~F}_{0} \times\left(\frac{\lambda}{\lambda_{0}}\right)^{+\beta} & \lambda<\lambda_{0}\end{array}\right.$,

where we use two power-law indices, $\alpha$ and $\beta$, to control the red and blue sides of the emission line profile, respectively. $\lambda_{0}$ is the peak wavelength of the emission line, and $F_{0}$ represents the peak intensity of the emission line. The ultraviolet lines are divided into two categories, high-ionization lines (HILs), including N v, O IV, N IV], $\mathrm{C}$ IV, and He II, and low-ionization lines (LILs), including Si II, Si IV, O III], Al II, Al III, Si III, and C III] (Collin-Souffrin \& Lasota 1988). We use the same power-law indices for lines in the same category. When fitting Ly $\alpha$, we adopt the same $\alpha$ as that for HILs, but leave parameter $\beta$ free due to the intergalactic medium absorption of its blue wing (Nagao et al. 2006).

We fit Ly $\alpha \lambda 1216$, N v $\lambda 1240$, Si II $\lambda 1263$, Si IV $\lambda 1398$, O IV $\lambda 1402$,

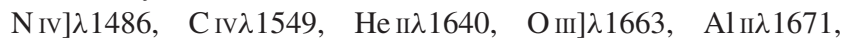
Al III $\lambda 1857$, Si III $\lambda 1887$, and C III] $\lambda 1909$ simultaneously and allow the shift of the central wavelength, $\lambda_{0}$, with respect to the rest-frame wavelength within $\pm 5 \AA$ corresponding to about $\pm 1000 \mathrm{~km} \mathrm{~s}^{-1}$. Unlike the multi-Gaussian profile, function 2 only has four parameters for each line. When deblending lines that are close to each other, the fewer free parameters we use, the fewer ambiguities we will encounter. A more detailed discussion of this method and comparison between power-law profile and Gaussian function was presented in Nagao et al. (2006). The fitting wavelength range for the emission lines is from 1210 to $2000 \AA$ for composite spectra in redshift 2.25 $3.75,1210$ to $1800 \AA$ for redshift $3.75-4.25$, and 1210 to $1600 \AA$ for redshift 4.25-5.25. The undefined feature in 1570-1631 $\AA$ which is called a ' $1600 \AA$ bump' is also excluded. The feature of $1600 \AA$ bump is ubiquitously presented in all our composite spectra and many previous studies also noticed this feature (Wilkes 1984; Boyle 1990; Laor et al. 1994; Nagao et al. 2006; Matsuoka et al. 2011). There still exists a debate on the interpretation of it. Nagao et al. (2006) have given a very detailed discussion on the $1600 \AA$ bump. One possibility is that the $1600 \AA$ bump is one of the C IV components because a very redshifted broad component for Ly $\alpha$ and $\mathrm{O}$ VI was found (Laor et al. 1994). Another possibility is that the $1600 \AA$ 

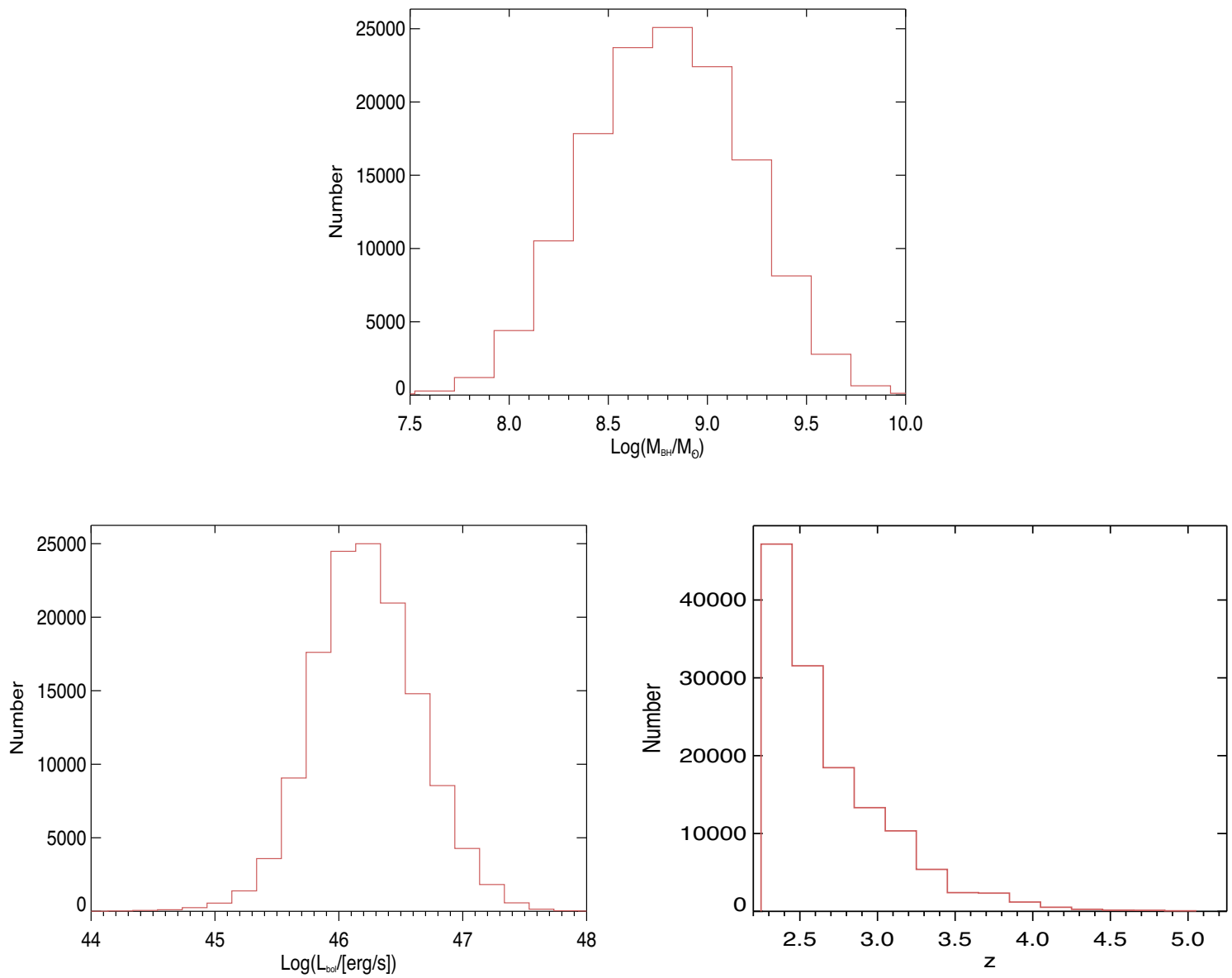

Figure 1. Histograms of our quasar sample selected from SDSS DR12. The redshift range is $2.25<z<5.25$ with the $\mathrm{BH}$ mass range $10^{7.5} \mathrm{M}_{\odot}<M_{\mathrm{BH}}<$ $10^{10} \mathrm{M}_{\odot}$, and bolometric luminosity range $10^{44.2} \mathrm{erg} \mathrm{s}^{-1}<L_{\mathrm{bol}}<10^{48} \mathrm{erg} \mathrm{s}^{-1}$. Upper panel: The $\mathrm{BH}$ mass distribution of our sample. There are only 86 quasars in $\mathrm{BH}$ mass range $10^{6.5} \mathrm{M}_{\odot}<M_{\mathrm{BH}}<10^{7.5} \mathrm{M}_{\odot}$ and 91 quasars in $\mathrm{BH}$ mass range $10^{10.0} \mathrm{M}_{\odot}<M_{\mathrm{BH}}<10^{10.6} \mathrm{M}_{\odot}$ and we exclude them. Lower left panel: The bolometric luminosity distribution of our sample. There are only seven quasars in bolometric luminosity range $10^{48.0} \mathrm{erg} \mathrm{s}^{-1}<L_{\mathrm{bol}}<10^{48.4} \mathrm{erg} \mathrm{s}^{-1}$ and 31 quasars in $10^{42} \mathrm{erg} \mathrm{s}^{-1}<L_{\mathrm{bol}}<10^{44.2} \mathrm{erg} \mathrm{s}^{-1}$ are excluded. Lower right panel: The histogram of the redshift of our sample.

Table 1. We divide quasar sample within redshift $2.25<z<4.25$ from SDSS DR12 into four redshift bins and each redshift bin contains $11 \mathrm{BH}$ mass bins. The number of the quasars in each bin is presented below. We exclude some bins due to the poor $\mathrm{S} / \mathrm{N}$ ratio of the composite spectra, and they are labelled with a ' $\%$.

\begin{tabular}{lcccc}
\hline $\log \left(M_{\mathrm{BH}} / \mathrm{M}_{\odot}\right) \backslash z$ & $2.25-2.75$ & $2.75-3.25$ & $3.25-3.75$ & $3.75-4.25$ \\
\hline $7.5-7.8$ & 419 & 119 & $/$ & $/$ \\
$7.8-8.0$ & 1462 & 468 & $/$ & $/$ \\
$8.0-8.2$ & 4584 & 1649 & 227 & $/$ \\
$8.2-8.4$ & 9249 & 3275 & 614 & 143 \\
$8.4-8.6$ & 14228 & 4849 & 1037 & 309 \\
$8.6-8.8$ & 17282 & 5628 & 1442 & 403 \\
$8.8-9.0$ & 16838 & 5473 & 1608 & 470 \\
$9.0-9.2$ & 13493 & 4707 & 1695 & 492 \\
$9.2-9.4$ & 7729 & 3276 & 1344 & 458 \\
$9.4-9.6$ & 2997 & 1542 & 707 & 309 \\
$9.6-10.0$ & 873 & 555 & 330 & 125 \\
\hline
\end{tabular}

bump is a blue-shifted component of the He II emission because Nagao et al. (2006) found a similar negative correlation between the flux of $1600 \AA / C$ IV with luminosity and $\mathrm{He}$ II/C IV with luminosity. The last possibility is that the $1600 \AA$ bump is caused by UV Fe II multiplet emission (Laor et al. 1994). Other heavy-blended lines
Table 2. We divide the quasar sample within redshift range $4.25<z<$ 5.25 into two redshift bins and each redshift bin contains two BH mass bins. The number of the quasars in each bin is presented below.

\begin{tabular}{lcc}
\hline $\log \left(M_{\mathrm{BH}} / \mathrm{M}_{\odot}\right) \backslash z$ & $4.25-4.75$ & $4.75-5.25$ \\
\hline $8.00-9.25$ & 254 & 65 \\
$9.25-10.00$ & 217 & 64 \\
\hline
\end{tabular}

such as $\mathrm{O}_{\text {I+ }} \mathrm{Si}$ II composite, $\mathrm{C}_{\text {III }}, \mathrm{N}_{\text {IV }}, \mathrm{Al}_{\text {II, }} \mathrm{N}_{\text {IIII }}$, and Fe II multiplets are also excluded in our fitting. To sum up, the wavelength regions, $1286-1357 \AA, 1570-1631 \AA$, and $1687-1833 \AA$, are excluded in our fitting process. Figs 2 and 3 are two typical examples of our fitting results. The upper panel shows the original composite spectrum, the best-fitting spectrum and the continuum. The lower panel shows the residual which is the ratio between the observed spectrum and the best fitting spectrum. The residual is in the range of $0.9-1.1$ in most of the cases suggesting that our line fitting results well describe the line profiles in our composite spectra.

As we have mentioned in the steps of generating composite spectra, we make 1000 'fake' composite spectra in the same BH mass (bolometric luminosity) and redshift bin and take their standard deviations as the uncertainties of the real composite spectra. Similarly, for the uncertainties of line ratios, we calculate 1000 line 
Table 3. We divide quasar sample within redshift $2.25<z<4.25$ from SDSS DR12 into four redshift bins and each redshift bin contains 15 bolometric luminosity bins. The number of the quasars in each bin is presented below. We exclude some bins due to the poor $\mathrm{S} / \mathrm{N}$ ratio of the composite spectra, and they are labelled with a ' $\%$. All the bins with bolometric luminosity $10^{44.2}-10^{44.6} \mathrm{erg} \mathrm{s}^{-1}$ are excluded due to the poor $\mathrm{S} / \mathrm{N}$ of their composite spectra, so the real bolometric luminosity range in this study is $10^{44.6}-10^{48.0} \mathrm{erg} \mathrm{s}^{-1}$.

\begin{tabular}{lcccc}
\hline $\log \left(L_{\text {bol }} /\left[\mathrm{erg} \mathrm{s}^{-1}\right]\right) \backslash z$ & $2.25-2.75$ & $2.75-3.25$ & $3.25-3.75$ & $3.75-4.25$ \\
\hline $44.6-45.0$ & 391 & $/$ & $/$ & $/$ \\
$45.0-45.2$ & 630 & 94 & $/$ & $/$ \\
$45.2-45.5$ & 3144 & 521 & $/$ & $/$ \\
$45.5-45.8$ & 12005 & 2353 & 217 & 37 \\
$45.8-46.0$ & 15569 & 4216 & 479 & 77 \\
$46.0-46.2$ & 17667 & 6122 & 1178 & 236 \\
$46.2-46.4$ & 15585 & 6161 & 1862 & 435 \\
$46.4-46.6$ & 11445 & 5053 & 202 & 660 \\
$46.6-46.8$ & 6908 & 3529 & 1521 & 583 \\
$46.8-47.0$ & 3541 & 1913 & 954 & 402 \\
$47.0-47.2$ & 1550 & 1004 & 433 & 196 \\
$47.2-47.4$ & 540 & 375 & 241 & 88 \\
$47.4-47.6$ & 125 & 130 & 78 & 31 \\
$47.6-48.0$ & 37 & 38 & 24 & 14
\end{tabular}

Table 4. We divide quasar sample within redshift $4.25<z<5.25$ from SDSS DR12 into two redshift bins and each redshift bin contains three bolometric luminosity bins. The number of the quasars in each bin is presented below.

\begin{tabular}{lcc}
\hline $\log \left(L_{\mathrm{bol}} /\left[\mathrm{erg} \mathrm{s}^{-1}\right]\right) \backslash z$ & $4.25-4.75$ & $4.75-5.25$ \\
\hline $46.0-46.5$ & 67 & 12 \\
$46.5-47.0$ & 274 & 65 \\
$47.0-47.5$ & 115 & 51 \\
\hline
\end{tabular}

ratios of these 1000 'fake' composite spectra and derive the standard deviations as their corresponding errors. These uncertainties take both the variance of individual spectra and systematics in the fitting procedure into account.

\subsection{Metallicity measurements}

Many studies have suggested that the broad emission-line flux ratios of quasars at the rest-frame ultraviolet and optical wavelength can provide accurate chemical abundance measurements in quasar BLRs (Hamann et al. 2002; Dietrich et al. 2003; Warner et al. 2003; Nagao et al. 2006; Matsuoka et al. 2011; Marziani et al. 2015; Sameshima, Yoshii \& Kawara 2017). The theoretical accuracy of any broad emission-line flux ratios as metallicity indicators depends on a variety of factors such as the central radiation field, temperature sensitivity of the emission-line ratio, the similarity of the ionization potentials and critical densities, and the extent to which the lineemitting regions overlap spatially (Hamann et al. 2002). In this work, we adopt two broad emission-line flux ratios to estimate the metallicity of quasar BLR: $\mathrm{N}$ v/C IV, (Si IV+O Iv])/C IV, which have been widely used in many previous studies (Hamann \& Ferland 1992; Hamann et al. 2002; Dietrich et al. 2003; Nagao et al. 2006; Juarez et al. 2009; Matsuoka et al. 2011; Wang et al. 2012). N v, C Iv, $\mathrm{Si}$ IV, and $\mathrm{O}$ IV] are ubiquitously presented in all the quasar spectra at redshift 2.25-5.25. Using the same diagnostics for all the composite spectra will prevent us from introducing systematic error. $\mathrm{N} v / \mathrm{C}$ IV can trace the chemical abundance based on the secondary nitrogen

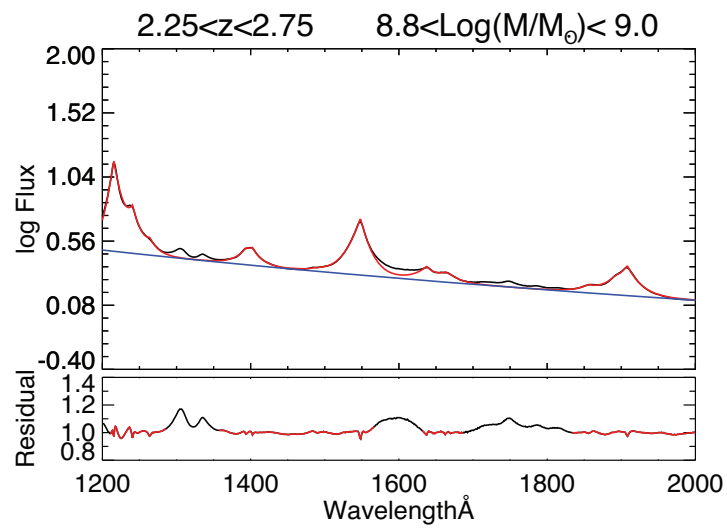

Figure 2. An example of our composite spectra at redshift $2.25-2.75$ in $\mathrm{BH}$ mass bin $10^{8.8}-10^{9.0} \mathrm{M}_{\odot}$. The upper panel shows the original composite spectrum (black solid line) and the fitting spectrum (red solid line). The blue solid line indicates the power-law fitting of the quasar continuum. The lower panel shows the residual which is the ratio of the composite spectrum and the fitting spectrum. The regions included in the line-fitting process are shown in red and the regions excluded are shown in black in the lower panel. The unit of the flux density is $10^{-17} \mathrm{erg} \mathrm{s}^{-1} \mathrm{~cm}^{-2} \AA^{-1}$.

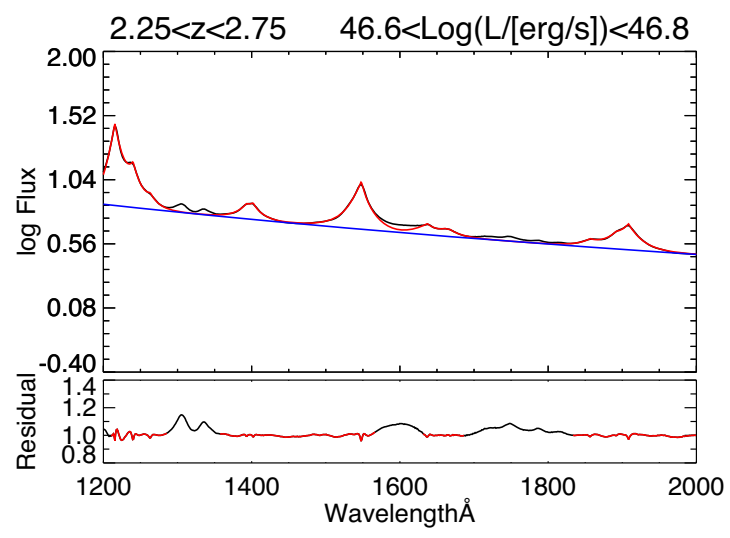

Figure 3. An example of our composite spectrum at redshift $2.25-2.75$ in bolometric luminosity bin $10^{46.6}-10^{46.8} \mathrm{erg} \mathrm{s}^{-1}$. The upper panel shows the original composite spectrum (black solid line) and the fitting spectrum (red solid line). The blue solid line indicates the power-law fitting of the quasar continuum. The lower panel shows the residual which is the ratio of the composite spectrum and the fitting spectrum. The regions included in the linefitting process are shown in red and the regions excluded are shown in black in the lower panel. The unit of the flux density is $10^{-17} \mathrm{erg} \mathrm{s}^{-1} \mathrm{~cm}^{-2} \AA^{-1}$.

theory which suggests that $\mathrm{N} / \mathrm{H}$ scales with $Z^{2}$ ( $Z$ in this paper is $\mathrm{O} / \mathrm{H})$. ( $\mathrm{Si}$ IV $\left.\left.+\mathrm{O}_{\mathrm{IV}}\right]\right) / \mathrm{C} \mathrm{IV}$, according to the simulation from Nagao et al. (2006), shows a significant correlation with BLR metallicity but not sensitive to the change of ionizing continuum. It traces the chemical abundance mainly because the relative importance of $\mathrm{C}$ IV as a coolant decreases as the BLR metallicity increases (Ferland et al. 1996; Juarez et al. 2009; Wang et al. 2012). We do not use line ratios relating $\mathrm{N}_{\mathrm{III}}$, $\mathrm{N}_{\mathrm{IV}}$ ], and $\mathrm{Si}$ II to estimate the metallicity because they are either highly blended with other strong lines or too weak to detect. We apply these two broad emission-line flux ratios to all the redshift bins from 2.25 to 5.25 .

We transfer the above two broad emission-line flux ratios into metallicities by using the simulation results based on photoionization models from Hamann et al. (2002) and Nagao et al. (2006). The relation between the metallicity and broad emission-line flux 
Table 5. The final metallicity $\left(Z / Z_{\odot}\right)$ of quasar BLR averaged from two different metallicity indicators in different BH mass bins at redshift 2.254.25 .

\begin{tabular}{lcccc}
\hline $\log \left(M_{\mathrm{BH}} / \mathrm{M}_{\odot}\right) \backslash z$ & $2.25-2.75$ & $2.75-3.25$ & $3.25-3.75$ & $3.75-4.25$ \\
\hline $7.5-7.8$ & $3.74_{-0.80}^{+0.62}$ & $3.59_{-0.74}^{+0.63}$ & $/$ & $/$ \\
$7.8-8.0$ & $3.67_{-0.69}^{+0.53}$ & $3.19_{-0.68}^{+0.54}$ & $/$ & $/$ \\
$8.0-8.2$ & $3.72_{-0.65}^{+0.49}$ & $3.29_{-0.64}^{+0.48}$ & $3.79_{-0.71}^{+0.58}$ & $/$ \\
$8.2-8.4$ & $3.92_{-0.67}^{+0.51}$ & $3.61_{-0.58}^{+0.52}$ & $3.80_{-0.67}^{+0.52}$ & $4.18_{-0.83}^{+0.67}$ \\
$8.4-8.6$ & $4.38_{-0.78}^{+0.57}$ & $4.07_{-0.77}^{+0.57}$ & $4.37_{-0.82}^{+0.61}$ & $4.44_{-0.84}^{+0.65}$ \\
$8.6-8.8$ & $4.91_{-1.04}^{+0.68}$ & $4.74_{-0.83}^{+0.68}$ & $5.24_{-0.96}^{+0.83}$ & $5.44_{-1.01}^{+0.86}$ \\
$8.8-9.0$ & $5.91_{-1.04}^{+0.88}$ & $5.91_{-1.14}^{+0.97}$ & $6.61_{-1.35}^{+1.25}$ & $6.99_{-1.45}^{+1.30}$ \\
$9.0-9.2$ & $7.43_{-1.52}^{+1.34}$ & $7.40_{-1.63}^{+1.46}$ & $8.43_{-1.83}^{+1.71}$ & $9.98_{-2.28}^{+2.28}$ \\
$9.2-9.4$ & $9.04_{-1.95}^{+1.82}$ & $9.06_{-2.13}^{+2.04}$ & $10.08_{-2.36}^{+2.34}$ & $11.32_{-2.75}^{+2.85}$ \\
$9.4-9.6$ & $10.65_{-2.38}^{+2.35}$ & $10.64_{-2.45}^{+2.43}$ & $12.02_{-2.99}^{+3.11}$ & $12.31_{-2.95}^{+3.12}$ \\
$9.6-10.0$ & $13.03_{-3.11}^{+3.26}$ & $13.46_{-3.32}^{+3.50}$ & $14.28_{-3.41}^{+3.68}$ & $20.19_{-4.84}^{+5.54}$ \\
\hline
\end{tabular}

ratio depends on the ionizing radiation field. Nagao et al. (2006) considered two possible spectral energy distribution (SED) models of the ionizing photons: one with a large UV thermal bump, and the other with a weak UV thermal bump. Hamann et al. (2002) considered three possible SED models which also span a wide range of possibilities from a strong 'big blue bump' to a simple power law with no bump at all. We considered all the five SED models from Nagao et al. (2006) and Hamann et al. (2002) for N v/C Iv. Hamann et al. (2002) did not calculate the case for $(\mathrm{Si} \mathrm{IV+O} \mathrm{IV])/C} \mathrm{IV,} \mathrm{so}$ we only considered two SED models from Nagao et al. (2006). (Si IV+O IV])/C IV almost stays the same (no more than $0.05 \mathrm{dex}$ change) with the change in SED (see fig. 29 in Nagao et al. 2006), suggesting the metallicity estimated from $(\mathrm{Si} \mathrm{IV+O} \mathrm{IV}]) / \mathrm{C}$ IV is not very sensitive to SED.

Some of our emission-line flux ratios indicate a metallicity greater than $10 \mathrm{Z}_{\odot}$ which has exceeded the upper limit of the simulation from Hamann et al. (2002) and Nagao et al. (2006). We assume that the relationship between emission-line flux ratio and the chemical abundance has the same trend when the metallicity is greater than $10 \mathrm{Z}_{\odot}$. We linearly extrapolate the points greater than $10 \mathrm{Z}_{\odot}$ in the log space. But it should be noticed that metallicity greater than 10 $\mathrm{Z}_{\odot}$ is not calibrated and potentially unphysical.

We calculate each metallicity by averaging the metallicity results from the considered different models. For the uncertainty ${ }^{2}$ of the metallicity, we take the highest metallicity derived from the considered SED models as the highest point of the error bar. The lowest points of the error bars are derived using the same way. Therefore, the metallicity errors in this paper take the systematic uncertainty introduced by different SED models into account. ${ }^{3}$

In order to combine the metallicity results from different metallicity indicators, we average the metallicity derived from (Si IV+O Iv])/C IV and $\mathrm{N} v / \mathrm{C}$ Iv. Tables $5-8$ present the final BLR

\footnotetext{
${ }^{2}$ We noticed that this is not a real uncertainty but an estimation of the metallicity range.

${ }^{3}$ The uncertainty of line measurement is usually very small, which is about 0.02 dex, comparing to the systematic error introduced by different SED models which can be up to $0.5 \mathrm{dex}$. But in some special cases, like the first blue point in the right-hand panel of Fig. 5, where the error is extremely large, it is due to poor fitting on emission line, not the uncertainty introduced by different SED models.
}

Table 6. The final metallicity $\left(Z / Z_{\odot}\right)$ of quasar BLR averaged from two different metallicity indicators in different BH mass bins at redshift 4.25 5.25 .

\begin{tabular}{lcc}
\hline $\log \left(M_{\mathrm{BH}} / \mathrm{M}_{\odot}\right) \backslash z$ & $4.25-4.75$ & $4.75-5.25$ \\
\hline $8.00-9.25$ & $3.66_{-0.74}^{+0.60}$ & $5.47_{-1.28}^{+1.39}$ \\
$9.25-10.00$ & $9.69_{-2.64}^{+2.81}$ & $8.89_{-2.35}^{+2.71}$ \\
\hline
\end{tabular}

Table 7. The final metallicity $\left(Z / Z_{\odot}\right)$ of quasar BLR averaged from two different metallicity indicators in different bolometric luminosity bins at redshift 2.25-4.25. All the bins with bolometric luminosity $10^{44.2}-10^{44.6} \mathrm{erg} \mathrm{s}^{-1}$ are excluded due to the poor $\mathrm{S} / \mathrm{N}$ of their composite spectra, so the real bolometric luminosity range in this study is $10^{44.6}-10^{48.0} \mathrm{erg} \mathrm{s}^{-1}$.

\begin{tabular}{lcccc}
\hline $\log \left(L_{\text {bol }} /\left[\mathrm{erg} \mathrm{s}^{-1}\right]\right) \backslash z$ & $2.25-2.75$ & $2.75-3.25$ & $3.25-3.75$ & $3.75-4.25$ \\
\hline $44.6-45.0$ & $3.38_{-0.65}^{+0.56}$ & $/$ & $/$ & $/$ \\
$45.0-45.2$ & $3.19_{-0.54}^{+0.41}$ & $4.01_{-0.85}^{+0.75}$ & $/$ & $/$ \\
$45.2-45.5$ & $3.45_{-0.61}^{+0.47}$ & $3.87_{-0.77}^{+0.65}$ & $/$ & $/$ \\
$45.5-45.8$ & $3.74_{-0.62}^{+0.46}$ & $3.58_{-0.68}^{+0.54}$ & $4.49_{-1.72}^{+1.92}$ & $6.42_{-1.03}^{+0.85}$ \\
$45.8-46.0$ & $4.30_{-0.73}^{+0.54}$ & $3.65_{-0.66}^{+0.49}$ & $4.75_{-0.85}^{+0.66}$ & $5.90_{-1.03}^{+1.03}$ \\
$46.0-46.2$ & $5.03_{-0.89}^{+0.66}$ & $3.90_{-0.72}^{+0.53}$ & $5.47_{-0.83}^{+0.69}$ & $7.05_{-1.31}^{+1.22}$ \\
$46.2-46.4$ & $5.83_{-0.97}^{+0.80}$ & $4.57_{-0.88}^{+0.66}$ & $6.15_{-1.05}^{+0.91}$ & $6.95_{-1.33}^{+1.23}$ \\
$46.4-46.6$ & $6.84_{-1.28}^{+1.13}$ & $5.27_{-0.96}^{+0.80}$ & $7.08_{-1.38}^{+1.26}$ & $7.65_{-1.50}^{+1.36}$ \\
$46.6-46.8$ & $7.86_{-1.60}^{+1.50}$ & $6.03_{-1.20}^{+1.04}$ & $8.26_{-1.76}^{+1.63}$ & $8.28_{-1.63}^{+1.51}$ \\
$46.8-47.0$ & $8.94_{-2.09}^{+2.02}$ & $6.64_{-1.44}^{+1.28}$ & $8.96_{-2.06}^{+2.02}$ & $10.41_{-2.50}^{+2.55}$ \\
$47.0-47.2$ & $10.22_{-2.51}^{+2.49}$ & $7.83_{-1.89}^{+1.82}$ & $10.02_{-2.45}^{+2.45}$ & $11.40_{-2.85}^{+3.00}$ \\
$47.2-47.4$ & $11.50_{-2.94}^{+3.01}$ & $7.76_{-1.86}^{+1.85}$ & $11.38_{-2.95}^{+3.10}$ & $11.36_{-2.74}^{+2.88}$ \\
$47.4-47.6$ & $13.02_{-3.53}^{+3.86}$ & $8.55_{-2.15}^{+2.19}$ & $12.06_{-3.27}^{+3.53}$ & $15.93_{-4.64}^{+6.13}$ \\
$47.6-48.0$ & $15.51_{-4.16}^{+4.77}$ & $10.32_{-2.74}^{+3.00}$ & $12.75_{-3.55}^{+4.16}$ & $14.51_{-3.82}^{+4.60}$ \\
\hline
\end{tabular}

Table 8. The final metallicity $\left(Z / Z_{\odot}\right)$ of quasar BLR averaged from two different metallicity indicators in different bolometric luminosity bins at redshift 4.25-5.25.

\begin{tabular}{lcc}
\hline $\log \left(L_{\text {bol }} /\left[\mathrm{erg} \mathrm{s}^{-1}\right]\right) \backslash z$ & $4.25-4.75$ & $4.75-5.25$ \\
\hline $46.0-46.5$ & $5.01_{-0.97}^{+0.92}$ & $4.43_{-1.39}^{+1.48}$ \\
$46.5-47.0$ & $6.89_{-1.58}^{+1.48}$ & $8.38_{-2.33}^{+2.53}$ \\
$47.0-47.5$ & $8.87_{-2.30}^{+2.48}$ & $9.15_{-2.12}^{+2.41}$ \\
\hline
\end{tabular}

metallicity in different redshift and BH mass (bolometric luminosity) bins along with error calculated from error propagation method.

\subsection{Results}

Fig. 4 shows the relation between the metallicity indicated from the two broad emission-line flux ratios and $\mathrm{BH}$ mass at different redshifts. Fig. 5 shows the relation between the metallicity estimated from the two broad emission-line flux ratios and bolometric luminosity at different redshifts. Two metallicity indicators all suggest that the metallicity of quasar BLR increases with BH mass and bolometric luminosity at all redshifts while no correlation between quasar BLR metallicity and redshift.

We examine the correlation between the metallicities derived from the above two broad emission-line flux ratios with $\mathrm{BH}$ mass, bolometric luminosity, or redshift by adopting a Spearman rankorder test. Tables 9, 10, and 11 present the Spearman rank-order 


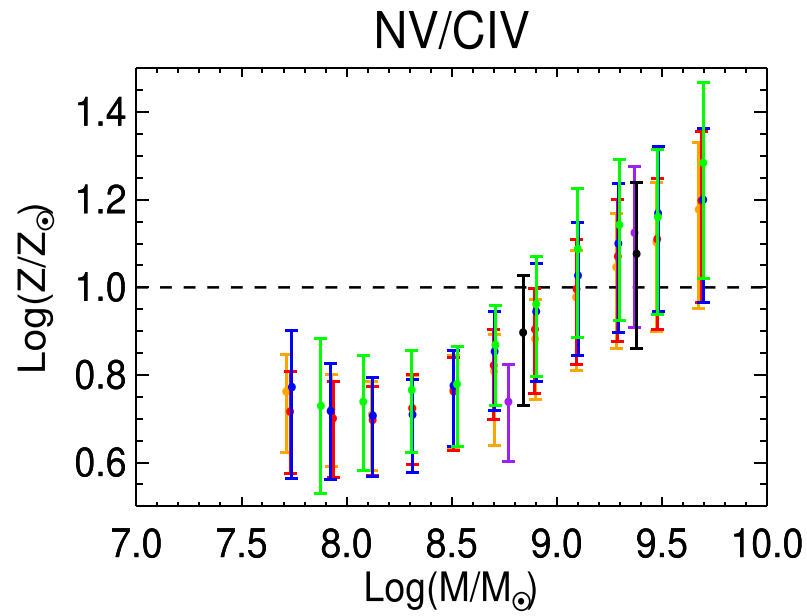

$(\mathrm{Si} \mathrm{IV+O} \mathrm{IV])/CIV}$

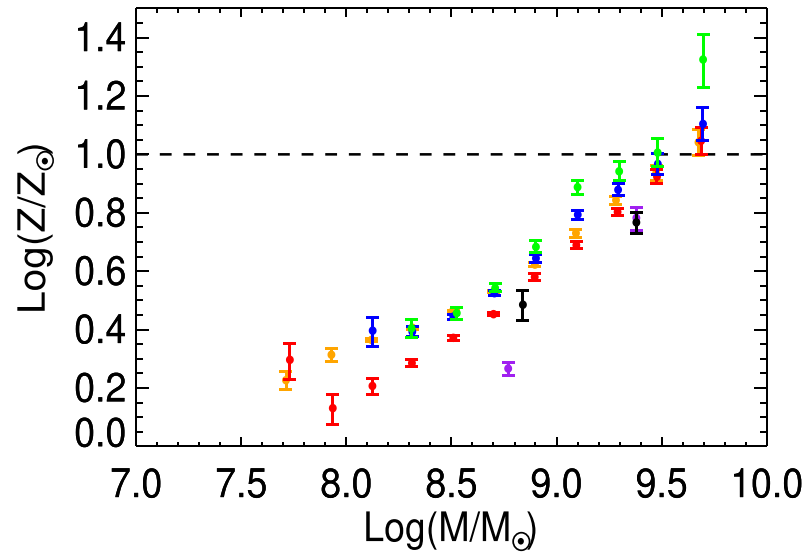

Figure 4. Metallicity of quasar BLR versus BH mass. The metallicity of quasar BLR is derived from two different metallicity indicators, which are labelled on the top of each panel. Orange, red, blue, green, purple, and black points represent the quasar BLR metallicity at redshifts 2.25-2.75, 2.75-3.25, 3.25-3.75, 3.75-4.25, 4.25-4.75, and 4.75-5.25. The dash lines indicate $10 \mathrm{Z}_{\odot}$. Metallicity greater than $10 \mathrm{Z}_{\odot}$ is uncertain.
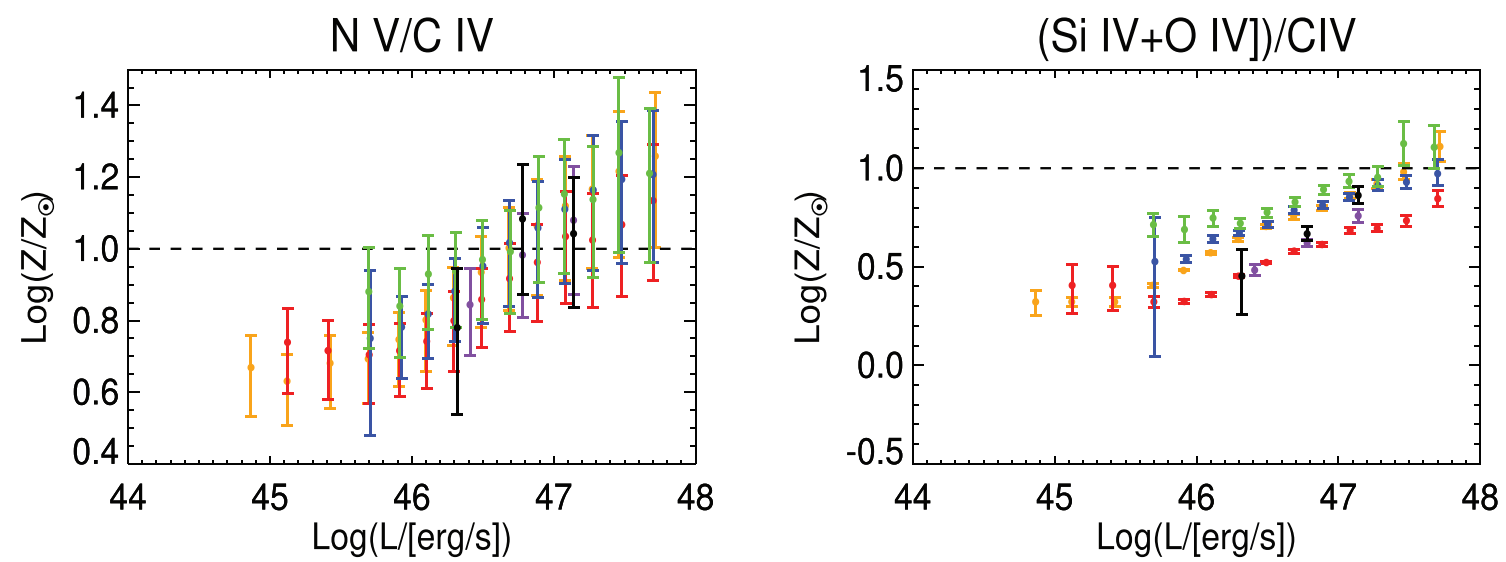

Figure 5. Metallicity of quasar BLR versus bolometric luminosity. The metallicity of quasar BLR is derived from two different metallicity indicators, which are labelled on the top of each panel. Orange, red, blue, green, purple, and black points represent the quasar BLR metallicity at redshifts 2.25-2.75, 2.75-3.25, $3.25-3.75,3.75-4.25,4.25-4.75$, and 4.75-5.25. The dash lines indicate $10 \mathrm{Z}_{\odot}$. Metallicity greater than $10 \mathrm{Z}_{\odot}$ is uncertain. The lowest bolometric luminosity point in redshift range 3.25-3.75 of ( $\mathrm{Si} \mathrm{IV}+\mathrm{O} \mathrm{IV}]$ )/C IV has a very large uncertainty due to the poor fitting of the emission line, not the systematic error from different SED models.

Table 9. The Spearman rank-order correlation coefficient $r_{\mathrm{s}}$ and the probability of the data being consistent with the null hypothesis that the metallicity is not correlated with $\mathrm{BH}$ mass, $\mathrm{p}\left(r_{\mathrm{s}}\right)$. The last row presents the Spearman rank-order test results of the relationship between final quasar BLR metallicity averaged from the two metallicity indicators and $\mathrm{BH}$ mass.

\begin{tabular}{lcc}
\hline Metallicity indicator & $r_{\mathrm{s}}$ & $\mathrm{p}\left(r_{\mathrm{s}}\right)$ \\
\hline (Si IV+O IV])/C IV & 0.87 & $7.5 \times 10^{-15}$ \\
$\mathrm{~N}$ v/C IV & 0.95 & $1.8 \times 10^{-23}$ \\
Average & 0.97 & $1.2 \times 10^{-26}$ \\
\hline
\end{tabular}

correlation coefficients $\left(r_{\mathrm{s}}\right)$ and the probability of the data being consistent with the null hypothesis that the metallicity is not correlated with $\mathrm{BH}$ mass, bolometric luminosity, and redshift $\left(\mathrm{p}\left(r_{\mathrm{s}}\right)\right)$, respectively. The larger the $r_{\mathrm{s}}$, the smaller the $\mathrm{p}\left(r_{\mathrm{s}}\right)$, the more significant the correlation is. The Spearman rank-order test shows that there are significant positive correlations between the metallicities derived from (Sirv+O IV])/C IV, N v/C IV, and BH mass. The Spearman
Table 10. The Spearman rank-order correlation coefficient $r_{\mathrm{s}}$ and the probability of the data being consistent with the null hypothesis that the metallicity is not correlated with bolometric luminosity, $\mathrm{p}\left(r_{\mathrm{s}}\right)$. The last row presents the Spearman rank-order test results of the relationship between final quasar BLR metallicity averaged from the two metallicity indicators and bolometric luminosity.

\begin{tabular}{lcc}
\hline Metallicity indicator & $r_{\mathrm{s}}$ & $\mathrm{p}\left(r_{\mathrm{s}}\right)$ \\
\hline (Si IV+O IV ] $) / \mathrm{C}_{\text {IV }}$ & 0.83 & $2.8 \times 10^{-15}$ \\
$\mathrm{~N}$ v/C IV & 0.81 & $8.1 \times 10^{-14}$ \\
Average & 0.92 & $1.9 \times 10^{-23}$
\end{tabular}

rank-order test also shows that there is a significant correlation between the metallicity derived from $(\mathrm{Si} \mathrm{IV}+\mathrm{O}$ IV $]$ )/C IV, N v/C IV with bolometric luminosity. There is no significant correlation between metallicity and redshift presented in any two metallicity indicators.

In order to illustrate the trend between quasar BLR metallicity, $\mathrm{BH}$ mass (bolometric luminosity), and redshift, we thus have to 
Table 11. The Spearman rank-order correlation coefficient $r_{\mathrm{s}}$ and the probability of the data being consistent with the null hypothesis that the metallicity is not correlated with redshift, $\mathrm{p}\left(r_{\mathrm{s}}\right)$. The last row presents the Spearman rank-order test results of the relationship between final quasar BLR metallicity averaged from the two metallicity indicators and redshift.

\begin{tabular}{lcc}
\hline Metallicity indicator & $r_{\mathrm{s}}$ & $\mathrm{p}\left(r_{\mathrm{s}}\right)$ \\
\hline (Si IV+O Iv])/C IV & 0.42 & $4.6 \times 10^{-3}$ \\
$\mathrm{~N}$ v/C IV & 0.23 & 0.14 \\
Average & 0.16 & 0.31 \\
\hline
\end{tabular}

average all the metallicities indicated from the two metallicity indicators as the final quasar BLR metallicity like we have mentioned before. The error of the final quasar BLR metallicity is calculated using error propagation. Fig. 6 shows the relationship of the final quasar BLR metallicity, BH mass (bolometric luminosity), and redshift. The metallicities of our quasar sample are super solar, ranging from 2.5 to $25.1 \mathrm{Z}_{\odot}$. They increase with $\mathrm{BH}$ mass and quasar luminosity. As mentioned before, the metallicity greater than $10 \mathrm{Z}_{\odot}$ is uncertain. Ferland \& Elitzur (1984) also suggested that a high metallicity in photoionized gas clouds may result in a very low equilibrium temperature, and consequently a very low emissivity of emission lines. We give a dash line in Figs $4-7$ to indicate the 10 $\mathrm{Z}_{\odot}$. Values that exceed this line are highly uncertain.

The Spearman rank-order test shows that there is a statistically significant correlation between the final quasar BLR metallicity and $\mathrm{BH}$ mass while no correlation with redshift. There is also a significant correlation between quasar BLR metallicity and bolometric luminosity, though, the correlation, compared to that of to $\mathrm{BH}$ mass, is weaker. This result is also consistent with many former researches (Dietrich et al. 2003; Warner et al. 2003; Nagao et al. 2006; Matsuoka et al. 2011). We also perform a linear fit, $y=\mathrm{k} x+b$, for the correlation between quasar BLR metallicity and BH mass, where $b=-2.37 \pm 0.18$ and $k=0.36 \pm 0.02$, and the correlation between quasar BLR metallicity and bolometric luminosity, where $b=-10.24 \pm 0.68$ and $k=0.24 \pm 0.01$ in Fig. 6 .

\section{DISCUSSION}

In this section, we compare the metallicities in quasar BLRs with those metallicities in their host galaxies. Due to the large brightness contrast between the quasar and their host galaxies, it is difficult to detect the rest-UV and/or optical metallicity diagnostic lines to measure their metallicity in high-redshift quasar host galaxies. In this study, we use the well-studied galaxy mass-metallicity relation (Erb et al. 2006; Maiolino et al. 2008; Zahid et al. 2013) to infer the metallicity in quasar host galaxies.

First, we convert the BH mass $\left(M_{\mathrm{BH}}\right)$ to host galaxy mass $\left(M_{\text {host }}\right)$ by adopting the evolution curve of $M_{\mathrm{BH}}: M_{\text {host }}$ ratio from Targett et al. (2012). These authors studied a sample of selected luminous $\left(M_{\mathrm{i}}<28\right)$ SDSS quasars at $z \simeq 4$ using AO observations. They estimated the stellar masses of their host galaxies using the evolutionary synthesis models of Bruzual \& Charlot (2003), assuming the initial mass function of Salpeter (1955), and using the C IV emission line to estimate the masses of their central SMBHs (see also Dong \& $\mathrm{Wu}$ 2016). The curve in fig. 6 of Targett et al. (2012) has combined the observational results at different redshifts from McLure et al. (2006), Peng et al. (2006), and Willott, McLure \& Jarvis (2003). We find that the quasar host galaxies in this study are in the stellar mass range of $10^{9}-10^{12} \mathrm{M}_{\odot}$, which spans a broad range of stellar mass. In order to infer the metallicities in the corresponding quasar host galaxies, we adopt the galaxy mass-metallicity relation at $z \sim 2.3$ from Zahid et al. (2013) and at $z \sim 3.5$ from Maiolino et al. (2008). We convert the metallicities in Maiolino et al. (2008) to Kobulnicky \& Kewley (2004) metallicity calibration (KK04), which is the same as in Zahid et al. (2013).

In Fig. 7, we compare the metallicities in the quasar BLRs and the metallicities in their host galaxies. We plot the quasar BLR metallicity as a function of their host galaxy mass. For a comparison, we also show the mass-metallicity relation of star-forming galaxies at $z \sim 2.3$ and 3.5. We find a significant discrepancy between the metallicities in quasar BLRs and their host galaxies. Tables 12 and 13 summarize the metallicity differences between the quasar BLRs and their host galaxies in different $\mathrm{BH}$ mass and redshift bins. We find the typical metallicities of the quasar BLRs are about 0.3-1.0 dex higher than those in their host galaxies, when taking the uncertainty of the $M_{\mathrm{BH}} / M_{\text {host }}$ into account. This discrepancy is quite intriguing, considering that it is well believed that the gas that feeds the central SMBH is provided by their host galaxies.

We also notice that there is an active debate on whether the $M_{\mathrm{BH}}: M_{\text {host }}$ ratio evolves with redshift. Some studies suggest that the redshift evolution seen in $M_{\mathrm{BH}}: M_{\text {host }}$ ratio is probably caused by the selection effect and there is no intrinsic redshift evolution in this ratio (Schulze \& Wisotzki 2011, 2014; Shen et al. 2015). Therefore, we also convert the BH mass to host galaxy mass by adopting a constant ratio $\sim 0.01-0.05$ from Targett et al. (2012). This conversion is made by converting the $\mathrm{BH}$ mass using a ratio of 0.03 (mid-point) and then using 0.01 and 0.05 to calculate the upper and lower limits of the estimated host galaxy mass. Tables 12 and 13 also summarize the metallicity discrepancy in this case. We found that the metallicity discrepancy is also about $0.3-1.0$ dex which is approximately the same when we assume that $M_{\mathrm{BH}}: M_{\text {host }}$ ratio is evolved with redshift. Therefore, the result is robust against the choice of the two cases.

We consider the following possibilities to explain the discrepancy of the metallicities in quasar BLRs and their host galaxies.

(i) The discrepancy between different metallicity diagnostic methods is a possible source to explain the discrepancy. There are three types of metallicity diagnostic methods: photoionization models, empirical calibrations, and direct- $\mathrm{T}_{\mathrm{e}}$ methods (Kewley \& Ellison 2008). It has been long known that the discrepancy of metallicities estimated from different strong-line metallicity diagnostic methods can be as large as 0.5 dex, particularly between the photoionization models and direct- $\mathrm{T}_{\mathrm{e}}$ method (e.g. Kewley \& Ellison 2008; Bian et al. 2017). The systematical uncertainty of metallicity estimated from different photoionization models can be up to 0.2 dex (e.g. Kewley \& Ellison 2008; Bian et al. 2017). Considering that the differences of metallicity between the quasar BLRs and their hosts are $0.3-0.8$ dex at $z=2.25-2.27$ and $0.4-1.0$ dex at $z=3.25-3.75$, it is unlikely that the different photoionization models cause such a large metallicity discrepancy between the quasar BLRs and their host galaxies.

The uncertainty in the metallicity calibration for BLR can be a source causing the metallicity discrepancy. The oversimplification of the secondary nitrogen might affect the estimation of chemical abundance. $\mathrm{H}$ II region studies show that secondary $\mathrm{N}$ production will dominate when $12+\log (\mathrm{O} / \mathrm{H})>8.3$ (Shields 1976; Henry, Edmunds \& Köppen 2000). It is obvious that the metallicity of quasar BLR has exceeded this value significantly, according to (Si IV+ $\mathrm{O}$ IV] $/ \mathrm{C}$ IV which does not depend on the secondary nitrogen production theory. Studies on quasar BLR metallicity also show that the metallicity measured using nitrogen emission lines corresponds 

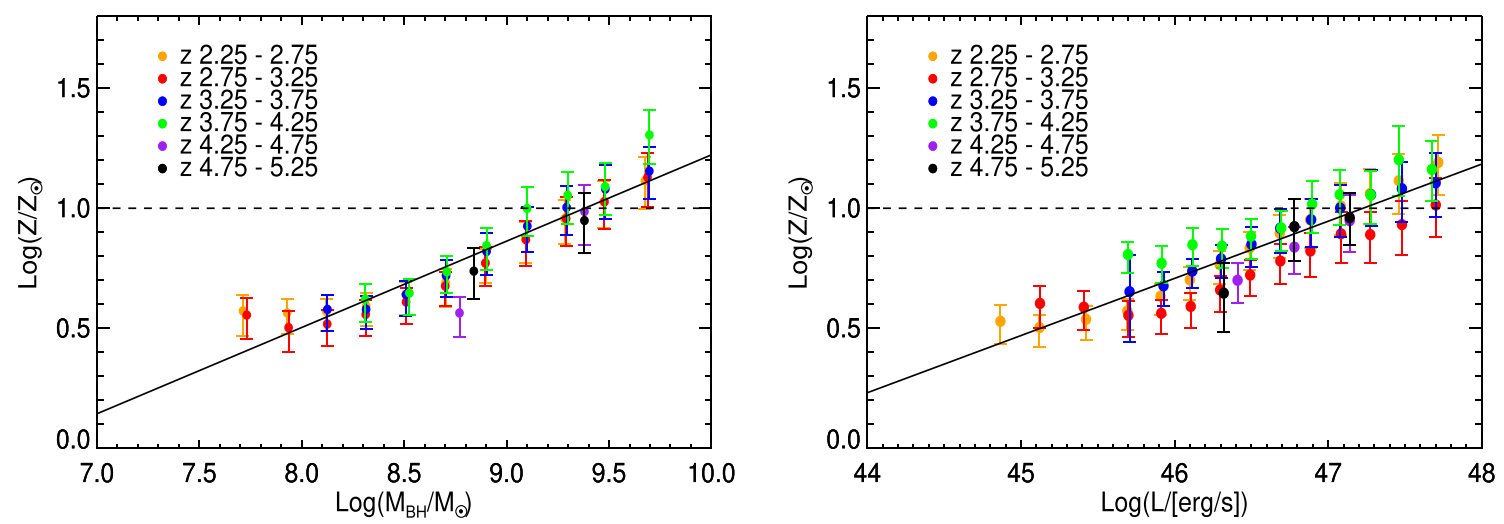

Figure 6. Orange, red, blue, green, purple, and black points represent the quasar BLR metallicity at redshifts 2.25-2.75, 2.75-3.25, 3.25-3.75, 3.75-4.25, 4.25-4.75, and 4.75-5.25. Left-hand panel: The relation between BH mass and the quasar BLR metallicity at different redshifts. We perform a linear fit, $y=$ $\mathrm{k} x+b$, for this relation where $b=-2.37 \pm 0.18$ and $k=0.36 \pm 0.02$. Right-hand panel: The relation between bolometric luminosity and the quasar BLR metallicity at different redshifts. We perform a linear fit, $y=\mathrm{k} x+b$, for this relation where $b=-10.24 \pm 0.68$ and $k=0.24 \pm 0.01$. Please see Tables 5-8 for the detailed metallicities and uncertainties shown on these two figures.

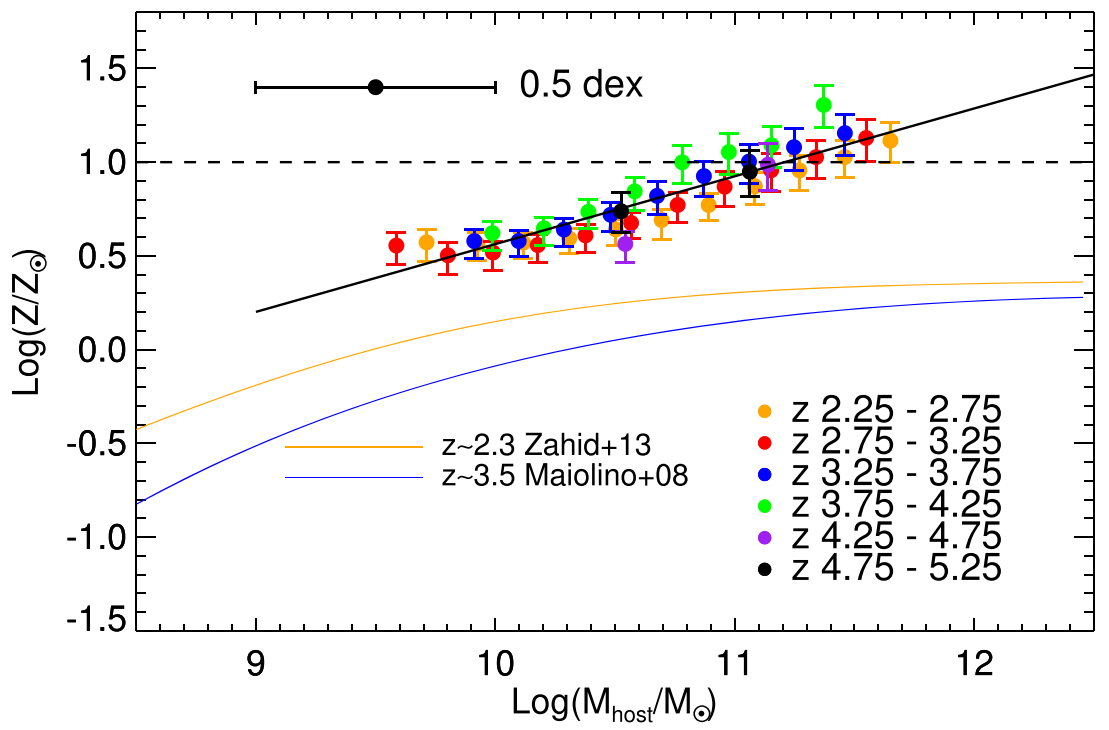

Figure 7. Comparison of the metallicity in quasar BLRs with that in their host galaxies inferred from star-forming galaxies. Orange, red, blue, green, purple, and black points correspond to the quasar BLR metallicity at redshifts $2.25-2.75,2.75-3.25,3.25-3.75,3.75-4.25,4.25-4.75$, and $4.75-5.25$ in different BH mass bins. The error bar on the left upper corner of the figure shows the typical value of the uncertainty of the estimated host galaxies masses introduced

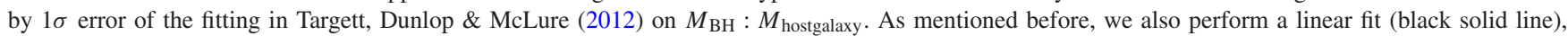
$y=\mathrm{k} x+b$, for the correlation between quasar BLR metallicity and BH mass where $b=-2.37 \pm 0.18$ and $k=0.36 \pm 0.02$. Orange solid line represents the mass-metallicity relationship of star-forming galaxies at $z \sim 2.3$ from Zahid et al. (2013). Blue solid line represents the mass-metallicity relationship of star-forming galaxies at $z \sim 3.5$ from Maiolino et al. (2008). The metallicity calibration of star-forming galaxies in this figure has all been transferred to KK04 calibration (Kobulnicky \& Kewley 2004). The dash line indicates $10 \mathrm{Z}_{\odot}$. Metallicity greater than $10 \mathrm{Z}_{\odot}$ is uncertain. For the detailed value of the comparison result between quasar BLR and host galaxy, please see Tables 12 and 13.

to that of using absorption line methods which is independent on the assumption of secondary N production (Hamann \& Ferland 1999; Hamann et al. 2002). Thus, it is reasonable for us to believe that secondary $\mathrm{N}$ production is prominent in quasar BLR (Hamann \& Ferland 1999). However, it is true that, based on some observational evidence (Garnett 1990; Pilyugin 1993; Marconi, Matteucci \& Tosi 1994; Pilyugin 1999), there is a scatter in N/O with a fixed $\mathrm{O} / \mathrm{H}$ caused by the bursts which temporarily lower N/O in the observed $\mathrm{H}$ II regions with sudden injections of fresh oxygen (Henry et al. 2000). Pagel (1985) suggests that there is an approximately \pm 0.3 dex uncertainty in $\mathrm{N} / \mathrm{O}$ at a fixed $\mathrm{O} / \mathrm{H}$ in the dwarf galaxies (Garnett 1990). We think that this cannot well explain the metallicity discrepancy between BLR and their hosts. Local turbulence in BLR cloud could be another source of uncertainty. The internal Doppler velocity $\left(v_{\mathrm{D}}\right)$ in BLR cloud is unknown (Bottorff et al. 2000). Calculation according to Hamann et al. (2002) suggested that change of $v_{\mathrm{D}}$ is not significant to affect the line ratio related to nitrogen. We refer the readers to their paper for more details on the calculation. 
Table 12. This table presents the masses of host galaxies transferred from BH masses, metallicities of quasar BLRs at redshift 2.25-2.75, metallicities in corresponding host galaxies at $z \sim 2.3$ from Zahid et al. (2013) in KK04 metallicity calibration and their differences $\Delta_{1} \log \left(Z / Z_{\odot}\right)$ (assuming $M_{\mathrm{BH}}: M_{\text {host }}$ evolves with redshift), $\Delta_{2} \log \left(Z / \mathrm{Z}_{\odot}\right)$ (assuming $M_{\mathrm{BH}}: M_{\text {host }}$ is constant) (Targett et al. 2012).

\begin{tabular}{lccccccccccc}
\hline $\log \left(M_{\mathrm{BH}} / \mathrm{M}_{\odot}\right)$ Median & 7.71 & 7.93 & 8.12 & 8.31 & 8.51 & 8.70 & 8.90 & 9.09 & 9.28 & 9.47 & 9.68 \\
\hline $\log \left(M_{\text {host }} / \mathrm{M}_{\odot}\right)$ & $9.71_{-0.49}^{+0.49}$ & $9.93_{-0.49}^{+0.49}$ & $10.12_{-0.49}^{+0.49}$ & $10.31_{-0.49}^{+0.49}$ & $10.50_{-0.49}^{+0.49}$ & $10.69_{-0.49}^{+0.49}$ & $10.89_{-0.49}^{+0.49}$ & $11.08_{-0.49}^{+0.49}$ & $11.27_{-0.49}^{+0.49}$ & $11.46_{-0.49}^{+0.49}$ & $11.65_{-0.50}^{+0.50}$ \\
$\log \left(Z_{\mathrm{BLR}} / \mathrm{Z}_{\odot}\right)$ & $0.57_{-0.10}^{+0.07}$ & $0.56_{-0.09}^{+0.06}$ & $0.57_{-0.08}^{+0.05}$ & $0.59_{-0.08}^{+0.05}$ & $0.64_{-0.09}^{+0.05}$ & $0.69_{-0.10}^{+0.06}$ & $0.77_{-0.08}^{+0.06}$ & $0.87_{-0.10}^{+0.07}$ & $0.96_{-0.11}^{+0.08}$ & $1.03_{-0.11}^{+0.09}$ & $1.11_{-0.12}^{+0.10}$ \\
$\log \left(Z_{\text {host }} / \mathrm{Z}_{\odot}\right)$ & $0.07_{-0.12}^{+0.09}$ & $0.13_{-0.10}^{+0.08}$ & $0.18_{-0.09}^{+0.06}$ & $0.21_{-0.07}^{+0.05}$ & $0.25_{-0.06}^{+0.04}$ & $0.27_{-0.05}^{+0.03}$ & $0.29_{-0.04}^{+0.03}$ & $0.31_{-0.03}^{+0.02}$ & $0.32_{-0.03}^{+0.02}$ & $0.33_{-0.02}^{+0.01}$ & $0.34_{-0.02}^{+0.01}$ \\
$\Delta_{1} \log \left(Z / \mathrm{Z}_{\odot}\right)$ & $0.50_{-0.20}^{+0.18}$ & $0.43_{-0.17}^{+0.16}$ & $0.39_{-0.15}^{+0.14}$ & $0.38_{-0.14}^{+0.13}$ & $0.39_{-0.13}^{+0.12}$ & $0.42_{-0.14}^{+0.11}$ & $0.48_{-0.11}^{+0.10}$ & $0.56_{-0.12}^{+0.11}$ & $0.63_{-0.12}^{+0.11}$ & $0.69_{-0.12}^{+0.11}$ & $0.77_{-0.13}^{+0.11}$ \\
$\Delta_{2} \log \left(Z / \mathrm{Z}_{\odot}\right)$ & $0.62_{-0.22}^{+0.21}$ & $0.53_{-0.19}^{+0.18}$ & $0.48_{-0.17}^{+0.17}$ & $0.45_{-0.16}^{+0.15}$ & $0.46_{-0.15}^{+0.14}$ & $0.47_{-0.15}^{+0.13}$ & $0.52_{-0.13}^{+0.12}$ & $0.59_{-0.13}^{+0.12}$ & $0.66_{-0.13}^{+0.12}$ & $0.71_{-0.13}^{+0.12}$ & $0.79_{-0.13}^{+0.12}$ \\
\hline
\end{tabular}

Table 13. This table presents the masses of host galaxies transferred from BH masses, metallicities of quasar BLRs at redshift 3.25-3.75, metallicities in corresponding host galaxies at $z \sim 3.5$ from Maiolino et al. (2008) in KK04 metallicity calibration, and their differences $\Delta_{1} \log \left(Z / \mathrm{Z}_{\odot}\right)\left(\right.$ assuming $M_{\mathrm{BH}}: M_{\text {host }}$ evolves with redshift), $\Delta_{2} \log \left(Z / \mathrm{Z}_{\odot}\right)$ (assuming $M_{\mathrm{BH}}: M_{\text {host }}$ is constant) (Targett et al. 2012).

\begin{tabular}{lccccccccc}
\hline $\log \left(M_{\mathrm{BH}} / \mathrm{M}_{\odot}\right)$ Median & 8.13 & 8.31 & 8.51 & 8.70 & 8.90 & 9.10 & 9.29 & 9.48 \\
\hline $\log \left(M_{\text {host }} / \mathrm{M}_{\odot}\right)$ & $9.99_{-0.53}^{+0.54}$ & $10.18_{-0.53}^{+0.54}$ & $10.38_{-0.53}^{+0.54}$ & $10.57_{-0.53}^{+0.54}$ & $10.76_{-0.53}^{+0.54}$ & $10.96_{-0.54}^{+0.54}$ & $11.15_{-0.54}^{+0.54}$ & $11.34_{-0.54}^{+0.54}$ & $11.55_{-0.54}^{+0.54}$ \\
$\log \left(Z_{\mathrm{BLR}} / \mathrm{Z}_{\odot}\right)$ & $0.58_{-0.09}^{+0.06}$ & $0.58_{-0.08}^{+0.06}$ & $0.64_{-0.09}^{+0.06}$ & $0.72_{-0.09}^{+0.06}$ & $0.82_{-0.10}^{+0.08}$ & $0.93_{-0.11}^{+0.08}$ & $1.00_{-0.12}^{+0.09}$ & $1.08_{-0.12}^{+0.10}$ & $1.15_{-0.12}^{+0.10}$ \\
$\log \left(Z_{\text {host }} / \mathrm{Z}_{\odot}\right)$ & $-0.12_{-0.13}^{+0.11}$ & $-0.06_{-0.12}^{+0.09}$ & $-0.003_{-0.10}^{+0.08}$ & $0.05_{-0.09}^{+0.07}$ & $0.09_{-0.08}^{+0.06}$ & $0.13_{-0.07}^{+0.06}$ & $0.16_{-0.06}^{+0.05}$ & $0.19_{-0.05}^{+0.04}$ & $0.21_{-0.05}^{+0.03}$ \\
$\Delta_{1} \log \left(Z / \mathrm{Z}_{\odot}\right)$ & $0.69_{-0.20}^{+0.19}$ & $0.64_{-0.18}^{+0.17}$ & $0.64_{-0.17}^{+0.16}$ & $0.67_{-0.16}^{+0.16}$ & $0.73_{-0.16}^{+0.16}$ & $0.80_{-0.16}^{+0.15}$ & $0.84_{-0.16}^{+0.15}$ & $0.89_{-0.17}^{+0.15}$ & $0.94_{-0.15}^{+0.15}$ \\
$\Delta_{2} \log \left(Z / \mathrm{Z}_{\odot}\right)$ & $0.74_{-0.20}^{+0.20}$ & $0.68_{-0.19}^{+0.18}$ & $0.68_{-0.18}^{+0.17}$ & $0.71_{-0.17}^{+0.16}$ & $0.76_{-0.17}^{+0.16}$ & $0.82_{-0.17}^{+0.16}$ & $0.86_{-0.17}^{+0.16}$ & $0.91_{-0.17}^{+0.16}$ & $0.95_{-0.16}^{+0.15}$ \\
\hline
\end{tabular}

i) There is also the possibility that host galaxies of quasars do not follow the average mass-metallicity relation of galaxies. The metallicity of galaxy depends not only on the stellar mass but also SFR. Studies show that host galaxies of luminous quasars are mergertriggered starburst galaxies and the SFR is about a few hundred to one thousandth $\mathrm{M}_{\odot} \mathrm{yr}^{-1}$ (Bertoldi et al. 2003; Wang et al. 2013). Wang et al. (2011a) observed nine $z \sim 6$ quasars and found that the average SFR of the $5 \mathrm{~mm}$-detected $m_{1450} \geq 20.2$ quasars is about $560 \mathrm{M}_{\odot} \mathrm{yr}^{-1}$. Dong \& Wu (2016) studied 207 quasars selected from SDSS quasar catalogues and the Herschel Stripe 82 survey and find that their SFRs are about $500 \mathrm{M}_{\odot} \mathrm{yr}^{-1}$. It is much larger than the SFR of UV-selected normal star-forming galaxies at $z \sim 2.3$ (Erb et al. 2006) and $z \sim 3.5$ (Maiolino et al. 2008), which are used to measure mass-metallicity relation. According to the fundamental metallicity relation which states that the metallicity of galaxies with lower SFR is higher than that of galaxies with higher SFR for a given stellar mass (Mannucci, Salvaterra \& Campisi 2011; Lara-López et al. 2013), the metallicity of quasar host galaxies should be less than or at least approximately equal to that of star-forming galaxies with the same stellar mass. This suggests that the metallicity of quasar host galaxy is probably overestimated based on the massmetallicity relation of normal star-forming galaxies, which makes the discrepancy even larger. Therefore, the fundamental metallicity relation cannot explain the metallicity discrepancy between the quasar BLRs and quasar host galaxies.

ii) The metallicity gradient in quasar host galaxies is another possibility to cause this discrepancy. Studies show that luminous quasars exist at the late stage of the major mergers (Hopkins et al. 2008; Glikman et al. 2015). Rich et al. (2012) studied the metallicity gradient in a sample of luminous infrared galaxies and found that the typical metallicity gradient in the late phase of the galaxy merger is about $0.02 \mathrm{dex} \mathrm{kpc}^{-1}$. If considering the compact size of the quasar host galaxy (e.g. $\sim 1 \mathrm{kpc}$; Venemans et al. 2017), the metallicity gradient of host galaxies is only no more than 0.05 dex. It could not explain such a difference between the metallicity of quasar BLRs and host galaxies.

iii) Another source of uncertainty is due to the BH mass estimation. This will affect our estimation on the stellar masses of quasar host galaxies. The reliability of the $\mathrm{C}$ IV line to reproduce the more reliable $\mathrm{H} \alpha$ or $\mathrm{H} \beta$-based $\mathrm{BH}$ mass estimates is not well established (Baskin \& Laor 2005; Shen et al. 2008; Shen \& Liu 2012; Runnoe et al. 2013). The main criticism is that the single-epoch $C$ Iv profiles do not generally represent the reverberating BLR because of the existence of a low-velocity core component and a blue excess to the C IV emission, both of which do not reverberate (Denney 2012). This is probably due to the contribution from an accretion disc wind (Sulentic et al. 2007; Richards et al. 2011), which results in a strong outflow or from a more distant narrow emission-line region (Konigl \& Kartje 1994; Murray et al. 1995; Proga, Stone \& Kallman 2000; Everett 2005; Gallagher et al. 2015). Due to the above scepticism, it is necessary for us to calibrate our BH mass. We calibrate our BH mass using two methods. The first method calibrates the $\mathrm{C}$ IV-based $\mathrm{BH}$ mass by using the trend of $\mathrm{C}$ IV-based $\mathrm{BH}$ mass $/ \mathrm{H} \alpha$ based BH mass versus $\mathrm{C}$ Iv blueshift (Coatman et al. 2016). The C IV blueshift $\left(\mathrm{km} \mathrm{s}^{-1}\right)$ is defined as $c \times(1549.48 \AA$-fitted central wavelength of $\mathrm{C}_{\mathrm{IV}}$ in the composite spectra)/1549.48 $\AA$ and it is about $90-800 \mathrm{~km} \mathrm{~s}^{-1}$ in this work. We might underestimate the $\mathrm{C}_{\mathrm{IV}}$ blueshift because the $\mathrm{Z}_{-} \mathrm{VI}$ is probably mostly determined by $\mathrm{C}$ IV or other luminous lines like $\mathrm{Mg}$ II. However, on average, the differences among Z_VI, Z_Mg II, and Z_PCA (the redshift determined by using principal component analysis) are small, i.e. $<20 \mathrm{~km} \mathrm{~s}^{-1}$ (Pâris et al. 2017). This will only bring $\sim 0.02$ dex difference to the $\mathrm{BH}$ mass estimation which will not significantly affect our result. The other method is calibrating the $\mathrm{C}$ IV-based $\mathrm{BH}$ mass with the peak ratio of $\lambda 1400$ (the definition of $\lambda 1400$ is $\mathrm{Si}$ IV+O IV]) and C IV (Runnoe et al. 2013). The first method shows that we probably underestimate the $\mathrm{BH}$ mass by $-0.2-0.3$ dex while the second method shows $-0.3-0.3$ dex which is quite consistent with the first one. 
However, considering this uncertainty, the discrepancy especially in the higher $\mathrm{BH}$ mass bins still exists.

iv) The possible change of quasar ionizing photon radiation field might also affect our results. In this work, we have considered different photoionization models covering a broad parameter space (Hamann et al. 2002; Nagao et al. 2006). In the most extreme case, we might underestimate the metallicity of the quasars in the lowest BH mass bin or overestimate the metallicity of the quasars at the highest $\mathrm{BH}$ mass bin by a factor of about 0.1 dex. However, even taking this effect into account, the mass-metallicity relationship still exists and it cannot explain the metallicity discrepancy.

v) Goodman \& Tan (2004) proposed that the fragmentation of quasar disc may result in supermassive star formation and the star will migrate inwards to the central BH. Jiang \& Goodman (2011) carried out a further two-dimensional simulation. Star formation on quasar disc is a very interesting possibility to explain this metallicity discrepancy. There are a few pieces of observational evidence in nearby quiescent galaxies, active Seyferts, and in our Galactic centre (Ghez et al. 2003; Lauer et al. 2005; Davies et al. 2007; Martins et al. 2008) suggesting that stellar discs do appear within a few parsec or even $\sim 10^{-2} \mathrm{pc}$ of the SMBH, but a direct observation to resolve the star formation on quasar disc is still lacking (Jiang $\&$ Goodman 2011). The luminous supermassive star will blend in the light of quasar. Goodman \& Tan (2004) and Jiang \& Goodman (2011) suggested that future periodicity searches or gravitational wave detections (Levin 2003) would bring more constraints on this topic. There are also multiple theories suggesting different scenarios as well. Levin $(2003,2007)$ and Collin \& Zahn (1999a,b) suggest that the fragmentation of the discs might result in the formation of many stars, even a nuclear stellar cluster, while Jiang \& Goodman (2011) suggest a single dominant mass. Besides the formation of the stars, whether the stars will eventually enrich the gas is another issue. A few theoretical studies show that the fragmentation of the unstable gaseous disc is able to give rise to the formation of protostars and consequently results in supernova explosion producing strong enriched outflows (Collin \& Zahn 1999b, 2008; Wang et al. 2011b). Another possible scenario is that if the formed stars exceed a few hundred solar masses, the stars may disrupt themselves immediately upon reaching the zero-age main sequence due to the pulsational instabilities and then enrich $\mathrm{CNO}$ abundance of the surrounding diffuse gas by returning the mass after disruption (Jiang \& Goodman 2011). However, there is also a possibility that the fragments may be accreted to the $\mathrm{BH}$ before contracting to a single dominant mass (Jiang \& Goodman 2011). Also, as suggested by Goodman \& Tan (2004), the migration time of the stars might be comparable to their main-sequence life time, so the stars might not be able to return their mass back to the gas by causing a supernova-like explosion, resulting in no metallicity enrichment. We cannot fully justify this possibility in this paper, and it is also likely that the metallicity discrepancy is caused by a combination effect of (1) to (5).

\section{CONCLUSION}

In this work, we used a large sample of quasar spectroscopic data from the SDSS DR12 with total $\sim 130000$ individual quasar spectra to investigate the metallicity of quasar BLR inferred from broad emission-line flux ratios based on photoionization models by fitting the composite spectra. The $\mathrm{BH}$ mass range and the bolometric luminosity range of the studied sample are $10^{7.5} \mathrm{M}_{\odot} \leq$ $M_{\mathrm{BH}} \leq 10^{10.0} \mathrm{M}_{\odot}$ and $10^{44.6} \mathrm{erg} \mathrm{s}^{-1} \leq L_{\text {bol }} \leq 10^{48.0} \mathrm{erg} \mathrm{s}^{-1}$, respectively. (Composite spectra in $10^{44.2} \mathrm{erg} \mathrm{s}^{-1} \leq L_{\text {bol }} \leq 10^{44.6} \mathrm{erg} \mathrm{s}^{-1}$ are excluded due to the poor S/N.) Our main result can be summarized as follows:

(i) The metallicity of quasar BLR ranges from 2.5 to $25.1 \mathrm{Z}_{\odot}$ inferred from broad emission-line ratios ( $\mathrm{N}$ v/C IV, ( $\mathrm{Si}$ IV+O IV)/C IV). Metallicity greater than $10 \mathrm{Z}_{\odot}$ is uncertain. There is a statistically significant correlation between quasar BLR metallicity and BH mass (bolometric luminosity), but the metallicity does not evolve with redshift.

(ii) We compared the metallicity of quasar BLR with that of host galaxies inferred from the mass-metallicity relation of star-forming galaxy and find that the metallicity of quasar BLRs is higher than their host galaxies by $0.3-1.0$ dex.

(iii) We considered several possibilities that cause the discrepancy, such as the effect of different SED models, systematic uncertainty of different metallicity diagnostic methods, mass-metallicity relations, C IV-based $\mathrm{BH}$ mass, the metallicity gradient in quasar hosts, and some other possibilities. However, none of the above possibilities can well explain the large metallicity difference between the quasar BLRs and quasar host galaxies.

(iv) We proposed that the origin of the metallicity from quasar BLRs and their hosts may be different. Star formation probably occurs on quasar accretion discs which enriches the gas close to the $\mathrm{BH}$ and may causes this discrepancy. However, there is no decisive observational evidence currently and the theory is also incomplete. Further studies are needed to justify this possibility.

\section{ACKNOWLEDGEMENTS}

We thank Fred Hamann and the anonymous referee for comments that significantly improved the work, and Tohru Nagao for useful discussions. FX gratefully acknowledges the support from the undergraduate research programme funding of Beijing Normal University. YS acknowledges support from an Alfred P. Sloan Research Fellowship and NSF grant AST-1715579. Funding for the SDSS IV has been provided by the Alfred P. Sloan Foundation, the U.S. Department of Energy Office of Science, and the Participating Institutions. SDSS-IV acknowledges support and resources from the Center for High Performance Computing at the University of Utah. The SDSS web site is www.sdss.org.

SDSS-IV is managed by the Astrophysical Research Consortium for the Participating Institutions of the SDSS Collaboration including the Brazilian Participation Group, the Carnegie Institution for Science, Carnegie Mellon University, the Chilean Participation Group, the French Participation Group, Harvard-Smithsonian Center for Astrophysics, Instituto de Astrofísica de Canarias, The Johns Hopkins University, Kavli Institute for the Physics and Mathematics of the Universe (IPMU)/ University of Tokyo, Lawrence Berkeley National Laboratory, Leibniz Institut für Astrophysik Potsdam (AIP), Max-Planck-Institut für Astronomie (MPIA Heidelberg), Max-Planck-Institut für Astrophysik (MPA Garching), Max-Planck-Institut für Extraterrestrische Physik (MPE), National Astronomical Observatories of China, New Mexico State University, New York University, University of Notre Dame, Observatário Nacional/MCTI, The Ohio State University, Pennsylvania State University, Shanghai Astronomical Observatory, United Kingdom Participation Group, Universidad Nacional Autónoma de México, University of Arizona, University of Colorado Boulder, University of Oxford, University of Portsmouth, University of Utah, University of Virginia, University of Washington, University of Wisconsin, Vanderbilt University, and Yale University. 


\section{REFERENCES}

Alam S. et al., 2015, ApJS, 219, 12

Asplund M., Grevesse N., Sauval A. J., Scott P., 2009, ARA\&A, 47, 481

Baskin A., Laor A., 2005, MNRAS, 356, 1029

Bennert N., Jungwiert B., Komossa S., Haas M., Chini R., 2006a, A\&A, 456, 953

Bennert N., Jungwiert B., Komossa S., Haas M., Chini R., 2006b, A\&A, 459,55

Bertoldi F., Carilli C. L., Cox P., Fan X., Strauss M. A., Beelen A., Omont A., Zylka R., 2003, A\&A, 406, L55

Bian F., Kewley L. J., Dopita M. A., Blanc G. A., 2017, ApJ, 834, 51

Bottorff M., Ferland G., Baldwin J., Korista K., 2000, ApJ, 542, 644

Boyle B. J., 1990, MNRAS, 243, 231

Bruzual G., Charlot S., 2003, MNRAS, 344, 1000

Cardelli J. A., Clayton G. C., Mathis J. S., 1989, ApJ, 345, 245

Coatman L., Hewett P. C., Banerji M., Richards G. T., 2016, MNRAS, 461, 647

Collin S., Zahn J.-P., 1999a, Ap\&SS, 265, 501

Collin S., Zahn J.-P., 1999b, A\&A, 344, 433

Collin S., Zahn J.-P., 2008, A\&A, 477, 419

Collin-Souffrin S., Lasota J.-P., 1988, PASP, 100, 1041

Davies R. I., Müller Sánchez F., Genzel R., Tacconi L. J., Hicks E. K. S., Friedrich S., Sternberg A., 2007, ApJ, 671, 1388

Denney K. D., 2012, ApJ, 759, 44

Dietrich M., Hamann F., Shields J. C., Constantin A., Heidt J., Jäger K., Vestergaard M., Wagner S. J., 2003, ApJ, 589, 722

Dong X. Y., Wu X.-B., 2016, ApJ, 824, 70

Du P., Wang J.-M., Hu C., Valls-Gabaud D., Baldwin J. A., Ge J.-Q., Xue S.-J., 2014, MNRAS, 438, 2828

Erb D. K., Shapley A. E., Pettini M., Steidel C. C., Reddy N. A., Adelberger K. L., 2006, ApJ, 644, 813

Everett J. E., 2005, ApJ, 631, 689

Fan X., Carilli C. L., Keating B., 2006a, ARA\&A, 44, 415

Fan X. et al., 2006b, AJ, 132, 117

Ferland G. J., Elitzur M., 1984, ApJ, 285, L11

Ferland G. J., Baldwin J. A., Korista K. T., Hamann F., Carswell R. F., Phillips M., Wilkes B., Williams R. E., 1996, ApJ, 461, 683

Gallagher S. C., Everett J. E., Abado M. M., Keating S. K., 2015, MNRAS, 451, 2991

Garnett D. R., 1990, ApJ, 363, 142

Ghez A. M. et al., 2003, ApJ, 586, L127

Glikman E., Simmons B., Mailly M., Schawinski K., Urry C. M., Lacy M., 2015, ApJ, 806, 218

Goodman J., Tan J. C., 2004, ApJ, 608, 108

Guo Y. et al., 2016, ApJ, 822, 103

Hamann F., Ferland G., 1992, ApJ, 391, L53

Hamann F., Ferland G., 1999, ARA\&A, 37, 487

Hamann F., Korista K. T., Ferland G. J., Warner C., Baldwin J., 2002, ApJ, 564,592

Henry R. B. C., Edmunds M. G., Köppen J., 2000, ApJ, 541, 660

Hopkins P. F., Hernquist L., Cox T. J., Kereš D., 2008, ApJS, 175, 356

Jiang L., Fan X., Vestergaard M., Kurk J. D., Walter F., Kelly B. C., Strauss M. A., 2007, AJ, 134, 1150

Jiang Y.-F., Goodman J., 2011, ApJ, 730, 45

Jones T., Stark D. P., Ellis R. S., 2012, ApJ, 751, 51

Juarez Y., Maiolino R., Mujica R., Pedani M., Marinoni S., Nagao T., Marconi A., Oliva E., 2009, A\&A, 494, L25

Kewley L. J., Ellison S. L., 2008, ApJ, 681, 1183

Kobulnicky H. A., Kewley L. J., 2004, ApJ, 617, 240

Konigl A., Kartje J. F., 1994, ApJ, 434, 446

Laor A., Bahcall J. N., Jannuzi B. T., Schneider D. P., Green R. F., Hartig G. F., 1994, ApJ, 420, 110

Lara-López M. A. et al., 2013, MNRAS, 434, 451

Lauer T. R. et al., 2005, AJ, 129, 2138

Levin Y., 2003, preprint (arXiv:astro-ph/0307084)

Levin Y., 2007, MNRAS, 374, 515

Ludwig R. R., Greene J. E., Barth A. J., Ho L. C., 2012, ApJ, 756, 51
Ly C., Malkan M. A., Nagao T., Kashikawa N., Shimasaku K., Hayashi M., 2014, ApJ, 780, 122

Maier C., Lilly S. J., Ziegler B. L., Contini T., Pérez Montero E., Peng Y., Balestra I., 2014, ApJ, 792, 3

Maiolino R. et al., 2008, A\&A, 488, 463

Mannucci F., Salvaterra R., Campisi M. A., 2011, MNRAS, 414, 1263

Marconi G., Matteucci F., Tosi M., 1994, MNRAS, 270, 35

Martins F., Gillessen S., Eisenhauer F., Genzel R., Ott T., Trippe S., 2008, ApJ, 672, L119

Marziani P., Sulentic J. W., Negrete C. A., Dultzin D., Del Olmo A., Martínez Carballo M. A., Zwitter T., Bachev R., 2015, Ap\&SS, 356, 339

Matsuoka K., Nagao T., Marconi A., Maiolino R., Taniguchi Y., 2011, A\&A, 527, A 100

McLure R. J., Jarvis M. J., Targett T. A., Dunlop J. S., Best P. N., 2006 , MNRAS, 368, 1395

Mortlock D. J. et al., 2011, Nature, 474, 616

Murray N., Chiang J., Grossman S. A., Voit G. M., 1995, ApJ, 451, 498

Nagao T., Marconi A., Maiolino R., 2006, A\&A, 447, 157

Nagao T., Maiolino R., Marconi A., Matsuoka K., Taniguchi Y., 2010, in Peterson B. M., Somerville R. S., Storchi-Bergmann T., eds, Proc. IAU Symp. 267, Co-Evolution of Central Black Holes and Galaxies. Kluwer, Dordrecht, p. 73

Onodera M. et al., 2016, ApJ, 822, 42

Pagel B. E. J., 1985, in Danziger I. J., Matteucci F., Kjar K., eds, European Southern Observatory Conference and Workshop Proceedings, Vol. 21, p. 155

Pâris I. et al., 2017, A\&A, 597, A79

Peng C. Y., Impey C. D., Rix H.-W., Kochanek C. S., Keeton C. R., Falco E. E., Lehár J., McLeod B. A., 2006, ApJ, 649, 616

Pilyugin L. S., 1993, A\&A, 277, 42

Pilyugin L. S., 1999, A\&A, 346, 428

Proga D., Stone J. M., Kallman T. R., 2000, ApJ, 543, 686

Rich J. A., Torrey P., Kewley L. J., Dopita M. A., Rupke D. S. N., 2012, ApJ, 753, 5

Richards G. T. et al., 2006, ApJS, 166, 470

Richards G. T. et al., 2011, AJ, 141, 167

Ross N. P. et al., 2012, ApJS, 199, 3

Runnoe J. C., Brotherton M. S., Shang Z., DiPompeo M. A., 2013, MNRAS, 434,848

Salim S., Lee J. C., Davé R., Dickinson M., 2015, ApJ, 808, 25

Salpeter E. E., 1955, ApJ, 121, 161

Sameshima H., Yoshii Y., Kawara K., 2017, ApJ, 834, 203

Sanders R. L. et al., 2015, ApJ, 799, 138

Schlegel D. J., Finkbeiner D. P., Davis M., 1998, ApJ, 500, 525

Schulze A., Wisotzki L., 2011, A\&A, 535, A87

Schulze A., Wisotzki L., 2014, MNRAS, 438, 3422

Shen Y., 2013, Bull. Astron. Soc. India, 41, 61

Shen Y., Liu X., 2012, ApJ, 753, 125

Shen Y., Greene J. E., Strauss M. A., Richards G. T., Schneider D. P., 2008, ApJ, 680, 169

Shen Y. et al., 2011, ApJS, 194, 45

Shen Y. et al., 2015, ApJ, 805, 96

Shields G. A., 1976, ApJ, 204, 330

Spergel D. N. et al., 2007, ApJS, 170, 377

Steidel C. C. et al., 2014, ApJ, 795, 165

Sulentic J. W., Bachev R., Marziani P., Negrete C. A., Dultzin D., 2007, ApJ, 666, 757

Targett T. A., Dunlop J. S., McLure R. J., 2012, MNRAS, 420, 3621

Vanden Berk D. E. et al., 2001, AJ, 122, 549

Venemans B. et al., 2017, ApJ, 837, 146

Vestergaard M., Peterson B. M., 2006, ApJ, 641, 689

Wang R. et al., 2011a, AJ, 142, 101

Wang H., Zhou H., Yuan W., Wang T., 2012, ApJ, 751, L23

Wang R. et al., 2013, ApJ, 773, 44

Wang J.-M. et al., 2011b, ApJ, 739, 3

Warner C., Hamann F., Dietrich M., 2003, ApJ, 596, 72

Wilkes B. J., 1984, MNRAS, 207, 73

Willott C. J., McLure R. J., Jarvis M. J., 2003, ApJ, 587, L15 
Wu X.-B. et al., 2015, Nature, 518, 512

Zahid H. J., Geller M. J., Kewley L. J., Hwang H. S., Fabricant D. G., Kurtz M. J., 2013, ApJ, 771, L19

Zheng W., Kriss G. A., Telfer R. C., Grimes J. P., Davidsen A. F., 1997, ApJ, 475, 469
Zuo W., Wu X.-B., Fan X., Green R., Wang R., Bian F., 2015, ApJ, 799, 189

This paper has been typeset from a $\mathrm{T}_{\mathrm{E}} \mathrm{X} / \mathrm{L} \mathrm{A} \mathrm{T}_{\mathrm{E}} \mathrm{X}$ file prepared by the author. 